\title{
Probing the many-body localization phase transition with superconducting circuits
}

\author{
Tuure Orell ${ }^{1},{ }^{1}$ Alexios A. Michailidis, ${ }^{2}$ Maksym Serbyn $\left(\mathbb{0},{ }^{2}\right.$ and Matti Silveri ${ }^{1}$ \\ ${ }^{1}$ Nano and Molecular Systems Research Unit, University of Oulu, 90014 Oulu, Finland \\ ${ }^{2}$ IST Austria, Am Campus 1, 3400 Klosterneuburg, Austria
}

(Received 9 July 2019; published 7 October 2019)

\begin{abstract}
Chains of superconducting circuit devices provide a natural platform for studies of synthetic bosonic quantum matter. Motivated by the recent experimental progress in realizing disordered and interacting chains of superconducting transmon devices, we study the bosonic many-body localization phase transition using the methods of exact diagonalization as well as matrix product state dynamics. We estimate the location of transition separating the ergodic and the many-body localized phases as a function of the disorder strength and the many-body on-site interaction strength. The main difference between the bosonic model realized by superconducting circuits and similar fermionic model is that the effect of the on-site interaction is stronger due to the possibility of multiple excitations occupying the same site. The phase transition is found to be robust upon including longer-range hopping and interaction terms present in the experiments. Furthermore, we calculate experimentally relevant local observables and show that their temporal fluctuations can be used to distinguish between the dynamics of Anderson insulator, many-body localization, and delocalized phases. While we consider unitary dynamics, neglecting the effects of dissipation, decoherence, and measurement back action, the timescales on which the dynamics is unitary are sufficient for observation of characteristic dynamics in the many-body localized phase. Moreover, the experimentally available disorder strength and interactions allow for tuning the many-body localization phase transition, thus making the arrays of superconducting circuit devices a promising platform for exploring localization physics and phase transition.
\end{abstract}

DOI: 10.1103/PhysRevB.100.134504

\section{INTRODUCTION}

Superconducting circuits, specifically arrays of superconducting transmon devices (Fig. 1), are a promising platform for quantum simulation. Transmon is a weakly anharmonic oscillator made of Josephson junctions and capacitors. However, scatter in circuit parameters, such as on-site energy and interaction strengths, is inevitable in the process of fabrication and hinders their applications [1]. Parameters of different circuit elements can be made also in situ tunable with microwave and magnetic flux controls, and disorder can be removed. This tunability requires extra junctions [2], engineered couplers [3], and control lines, which all add to device, characterization, and measurement complexity. Moreover, these ingredients add decoherence and dissipation and thus are not the most advantageous methods for large arrays.

The presence of intrinsic disorder suggests that transmon arrays can be a natural platform to study physics emergent from interplay between disorder and interactions. Sufficiently strong disorder may lead to a many-body localized phase-a stable phase of matter characterized by the breakdown of thermalization [4-6]. Many-body localized systems are characterized by a logarithmically slow entanglement spreading [7] and relaxation to a nonthermal state that retains memory of initial conditions. This is in contrast to ballistic spreading of entanglement in thermalizing systems that achieve thermal equilibrium and lose memory of the initial state [8]. The characteristic slow dynamics of the many-body localized phase makes it a prospective target for quantum simulators that are characterized by slow loss of coherence [9-11].
Many-body localization is an active research field at the intersection of nonequilibrium quantum dynamics, quantum thermalization, condensed matter physics, quantum information, computational physics, and other fields [4-6]. The major focus of earlier studies was on localization in one-dimensional systems with the two-dimensional local Hilbert space. Specific examples of such systems include interacting spinless fermions [12-14], spin-1/2 chains [15-19], and hard-core bosons [20] on one-dimensional lattices. In contrast, localization in bosonic systems received relatively little attention, with an exception of a few works [21-26]. The bosonic systems are more challenging for numerical studies [23], since the size of the local Hilbert space in a bosonic model with particle conservation is limited only by the total number of excitations in the system. This implies that the scaling of the total Hilbert space with system size heavily depends on the filling factor. Furthermore, as we show below, the disorder-dependent asymmetry in the density of states presents additional complications.

Recent research considered interacting microwave photons in disordered $[27,28]$ and clean $[29,30]$ superconducting circuit chains as well as bosonic $\mathrm{Rb}$ atoms in optical lattices [31-33] and demonstrated that bosonic systems provide good experimental platforms to study many-body physics. Motivated by this experimental progress with bosonic synthetic matter, we study here a chain of superconducting transmon devices (Fig. 1) realizing a disordered Bose-Hubbard model with attractive interactions [29,34] (Sec. II).

After introducing the model, we proceed with numerical calculation of the phase diagram, identifying the location of the phase transition that separates ergodic and many-body 


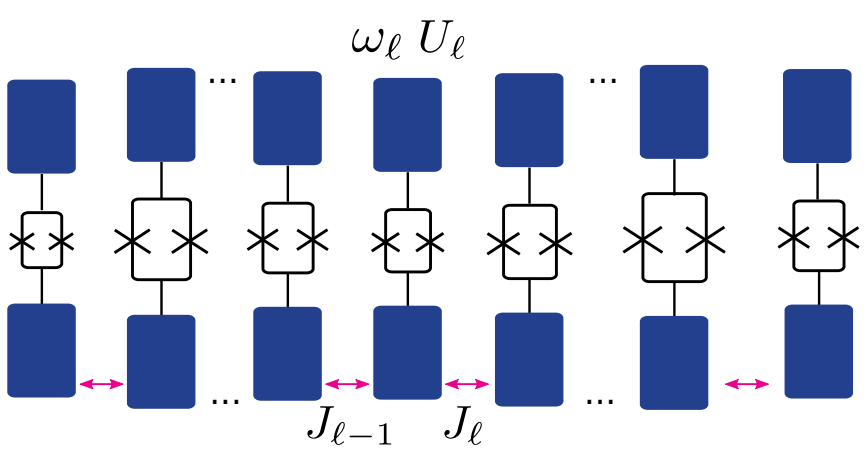

FIG. 1. Schematic of a transmon chain realizing the BoseHubbard model with attractive interactions. A transmon is made of Josephson junctions (black crosses) and capacitor plates (blue rectangles). Transmons are anharmonic oscillators with natural frequency $\omega$ and anharmonicity $U$ and they interact with each other through capacitive interaction $J$. Nonidentical sites visualize fabrication scatter and disorder.

localized phases as a function of disorder strength and interactions (Sec. III). In addition, we consider experimentally relevant longer range hopping and interaction terms in the Hamiltonian and study their effect on the many-body localization phase transition. Crucially, we demonstrate that the phase transition stays within the experimentally accessible range of parameters in the transmon chains.

In the second half of the paper, we consider time dynamics of local observables after a quench from an initial product state (Sec. IV). We show that the fluctuations of local observables can be used to experimentally distinguish between the dynamics of Anderson insulator, many-body localization, and delocalized phases on the experimentally accessible timescales. In other words, temporal fluctuations of local observables can serve as an alternative to the challenging measurement of the entanglement dynamics in a quantum quench [33,35]. Finally, we conclude with discussing future directions and possible effects arising from dephasing, dissipation, and measurement back action (Sec. V).

In addition to our two main results, the phase diagram of the bosonic Hubbard model and dynamics of local observables, our work extends the numerical methods for studies of many-body localization to bosonic systems. We utilize methods of exact diagonalization for solving eigenstates and resolving the phase diagram. Dynamics are performed using Krylov subspace methods [36] as well as time evolving block decimation [37], which is a Trotter-based evolution scheme for matrix product states [38]. As bosonic systems are rather an untrodden path in the context of numerics of interacting and disordered systems, we also present details on the used numerical methods and their scalability (Appendixes A-D). In particular, we discuss the combination of the $L D L$ matrix decomposition and the stochastic Chebyshev series expansion [39] for efficient estimation of the density of states without solving the full energy eigenvalue spectrum.

\section{TRANSMON ARRAY}

Transmon is a weakly anharmonic electric oscillator with natural frequency $\omega=\left(\sqrt{8 E_{\mathrm{C}} E_{\Sigma \mathrm{J}}}-E_{\mathrm{C}}\right) / \hbar$ made of ca- pacitor plates that set the capacitive charging energy $E_{\mathrm{C}}$ and Josephson junctions that act as a weakly nonlinear inductor $[2,40]$, setting the total Josephson energy $E_{\Sigma \mathrm{J}}$. In the transmon regime, the Josephson energy dominates over the charging energy, characterized by the ratio $E_{\Sigma \mathrm{J}} / E_{\mathrm{C}}$, in the range of 25-100. In this work, we are interested in probing many-body dynamics in a chain of transmons. The transmon chain is schematically depicted in Fig. 1, assuming transmons with a three-dimensional (3D) architecture [34,40] placed in a $3 \mathrm{D}$ cavity or a waveguide (not shown). The 3D architecture offers benefits in terms of low dissipation and decoherence rates as well as versatile geometric options in coupling of the transmons. In contrast, transmons with a two-dimensional (2D) architecture coupled to 2D cavities made on coplanar waveguide resonators [27,30] have very accurate, fast, and developed measurement and controlling schemes. However, both architectures are essentially similar from the point of view of unitary many-body dynamics.

Given the recent experimental demonstrations with chains and clusters of 10-20 superconducting qubits [27,30,41,42], the arrays are expected to reach 10-100 sites in the near future. Transmons interact with each other via capacitive dipole-dipole interaction $J$, whose strength can be tailored from 10 to $100 \mathrm{MHz}$ by changing the orientation and size of transmon capacitors [29,34]. The interaction strength should be compared with the dissipation and decoherence rates of the state-of-the-art devices [40,43-48]. The decoherence rates $\Gamma_{2}$ range from $10 \mathrm{kHz}$ in $3 \mathrm{D}$ architecture transmons to $25 \mathrm{kHz}$ in $2 \mathrm{D}$ architecture transmons. Similarly, the dissipation rates $\Gamma_{1}$ range from 2 to $5 \mathrm{kHz}$ in $3 \mathrm{D}$ and $2 \mathrm{D}$ architectures, respectively. Almost three orders of magnitude difference between the interaction strength and dissipation and decoherence rates yields an ample time frame of unitary many-body dynamics before disruptive dissipation and decoherence effects become important.

In quantum computing applications, one uses the transmon anharmonicity $U=E_{\mathrm{C}} / \hbar$ in the order of a few $100 \mathrm{MHz}[40,49]$ to turn transmons into effective two-level systems [50]. However, in the quantum simulations of the many-body physics, the higher energy levels and their bosonic excitation statistics can be accessed. In this case, the anharmonicity acts as an on-site interaction between bosonic excitations. Superconducting transmon devices can be easily driven with single-site accuracy, offering a possibility to study driven-dissipative dynamics [51]. Furthermore, superconducting qubit readout can be made almost perfectly quantum nondemolition [52,53], offering a possibility to explore manybody dynamics and entanglement phase transitions [54-59] under continuous repeated or variable strength [60] measurements.

In this section, we introduce the nondisordered BoseHubbard model realized by a chain of identical trasmons. In addition, we discuss additional terms present in the Hamiltonian - the longer range and higher order multiparticle interactions as well as transmon-specific disorder potential. As our main focus lies in many-body localization induced by the presence of strong disorder, we continue by discussing different ways to realize in situ tunable disorder and calculate the resulting many-body eigenspectrum. 
TABLE I. Experimentally relevant values for the parameters in the disordered Bose-Hubbard Hamiltonian (8). The dimensionless ratios are in the range $U / J=2-30$ and $W / J=0.1-200[27,29,30]$, which we demonstrate to be sufficient for tuning the many-body localization phase transition.

\begin{tabular}{lcc}
\hline \hline Parameter & Symbol & Value \\
\hline On-site energy & $\omega / 2 \pi$ & $5-10 \mathrm{GHz}$ \\
On-site interaction & $U / 2 \pi$ & $200-300 \mathrm{MHz}$ \\
Hopping & $J / 2 \pi$ & $10-100 \mathrm{MHz}$ \\
Disorder amplitude & $W / 2 \pi$ & $10 \mathrm{MHz}-2 \mathrm{GHz}$ \\
Transmon asymmetry & $d$ & 0.1 \\
\hline \hline
\end{tabular}

\section{A. Clean Bose-Hubbard model}

One-dimensional array of $L$ identical transmons (Fig. 1) is described by the attractive Bose-Hubbard model [27,29,30]

$$
\begin{aligned}
\hat{H}_{\mathrm{BH}} / \hbar= & \sum_{\ell=1}^{L} \omega \hat{n}_{\ell}-\sum_{\ell=1}^{L} \frac{U}{2} \hat{n}_{\ell}\left(\hat{n}_{\ell}-1\right) \\
& +\sum_{\ell=1}^{L-1} J\left(\hat{a}_{\ell}^{\dagger} \hat{a}_{\ell+1}+\hat{a}_{\ell} \hat{a}_{\ell+1}^{\dagger}\right),
\end{aligned}
$$

where $\hat{a}_{\ell}$ and $\hat{a}_{\ell}^{\dagger}$ are the bosonic annihilation and creation operators of the site $\ell,\left[\hat{a}_{\ell}, \hat{a}_{k}^{\dagger}\right]=\delta_{\ell, k}$, and $\hat{n}_{\ell}=\hat{a}_{\ell}^{\dagger} \hat{a}_{\ell}$ is the corresponding number operator. In the many-body language, the natural frequency $\omega$ corresponds to the on-site energy, capacitive dipole-dipole interaction $J$ is interpreted as the nearest-neighbor hopping between adjacent sites, and the anharmonicity $U=E_{\mathrm{C}} / \hbar$ serves as the on-site interaction. The Hamiltonian (1) conserves the total number of excitations since it commutes with the total occupation operator $\hat{N}=\sum_{\ell=1}^{L} \hat{n}_{\ell}$. It is therefore sufficient to study only a single subspace of the Hamiltonian with a fixed total number of excitations $N$. Here we focus mainly on the chain at halffilling, i.e., $N=L / 2$, with filling factor $f=N / L=1 / 2$. The experimentally relevant parameters are summarized in Table I.

We note that the on-site interaction is attractive. It stems from the cosine potential of the Josephson junctions, $E_{\mathrm{J}} \cos \left[\sqrt[4]{2 E_{\mathrm{C}} / E_{\Sigma \mathrm{J}}}\left(\hat{a}+\hat{a}^{\dagger}\right)\right]$, that softens as a function of the excitation number. The anharmonic term $U=E_{\mathrm{C}} / \hbar$ is the lowest order correction to the harmonic potential, and as the occupation number increases one has to take into account also higher order corrections, the first of such being

$$
\hat{H}_{\mathrm{HA}} / \hbar=\sum_{\ell=1}^{L} \frac{U_{2}}{6} \hat{n}_{\ell}\left(\hat{n}_{\ell}-1\right)\left(\hat{n}_{\ell}-2\right) .
$$

Here, the higher order anharmonicity, which is repulsive with $U_{2} / 2 \pi$ in the range $10-30 \mathrm{MHz}$, effectively reduces total anharmonicity of the transmon. The cosine potential also implies that there exists a finite number of bound states [61-63], denoted as the transmon states, whose total number per site is approximately $\sqrt{E_{\mathrm{J}} / E_{\mathrm{C}}}$. Because the excitations in this system are bosons, there is a possibility that they all occupy the same site. These facts introduce a theoretical upper limit for the validity of our model. Depending on the parameters, only $\sqrt{E_{\mathrm{J}} / E_{\mathrm{C}}} \sim 10$ lowest states of each transmon are bound.
Higher occupations on single sites break the Bose-Hubbard approximation, limiting the validity of our model to roughly 20 transmons at half-filling.

In addition to the nearest-neighbor interaction, in realistic systems there exists also longer range tunneling process between sites further apart. Tunneling between next-nearest neighbors is described by term

$$
\hat{H}_{\mathrm{LR}} / \hbar=\sum_{\ell=1}^{L-2} J_{2}\left(\hat{a}_{\ell}^{\dagger} \hat{a}_{\ell+2}+\hat{a}_{\ell} \hat{a}_{\ell+2}^{\dagger}\right) .
$$

The next-nearest neighbor hopping is typically weak compared to the nearest neighbor hopping, $J_{2} \sim J / 10$ [29]. Notice that the additional perturbations of Eqs. (2) and (3) also conserve the total number of excitations.

At zero temperature, the clean Bose-Hubbard Hamiltonian (1) can undergo a phase transition between the Mottinsulating and superfluid phases [64]. This is a groundstate phase transition studied in the repulsive Bose-Hubbard model. In this work, we are interested in the highly excited or infinite-temperature eigenstates of the attractive BoseHubbard model. Therefore, it is important to understand the structure of the many-body eigenspectrum and especially how it is affected by the on-site interaction $U$. We show the full energy spectrum of Eq. (1) with $L=10$ at half-filling in Fig. 2 for three values of the on-site interaction $U / J=0$ (a), 3.5 (b), and 20 (c). When discussing the energies of excited many-body eigenstates (shown in Fig. 2 as horizontal lines), it is often convenient to consider the normalized energy

$$
\epsilon=\frac{E-E_{\min }}{E_{\max }-E_{\min }} \in[0,1],
$$

where $E_{\min }$ and $E_{\max }$ are the smallest and largest eigenvalues of the Hamiltonian in the studied sector and $E \in\left[E_{\min }, E_{\max }\right]$ is an arbitrary energy eigenvalue.

In the absence of on-site interaction $U$, the Bose-Hubbard Hamiltonian describes a chain of coupled harmonic oscillators. This system has a symmetric spectrum [Fig. 2(a)], but as the anharmonicity is increased in Figs. 2(b) and 2(c), the symmetry is removed and the eigenstates begin to form minibands. Because of the negative anharmonicity, two excitations occupying the same transmon have smaller energy than two excitations on different sites. The lowest energies are obtained when all excitations occupy the same transmon. This is seen in the expectation values of the total anharmonicity operator $\sum_{\ell} \hat{n}_{\ell}\left(\hat{n}_{\ell}-1\right)$ (black dots in Fig. 2): The larger the expectation value, the more bosons are occupying a single site. This behavior is visible in Figs. 2(b) and 2(c), showing that in systems with nonzero $U$ the expectation value decreases as the energy is increased. In the limit of large $U / J$, the minibands are fully formed, the total anharmonicity is conserved within each band separately, and the nearest-neighbor hopping interaction weakly lifts the degeneracy within the bands.

To calculate the many-body spectrum, we utilize exact diagonalization of Hamiltonian (1). Exact numerical calculations of many-body quantum systems are very demanding due to the exponential scaling of the Hilbert space dimension. In our case, we are interested in $L$ coupled $(N+1)$-level systems with a total Hilbert space dimension $D_{L}=(N+1)^{L}$. Because 

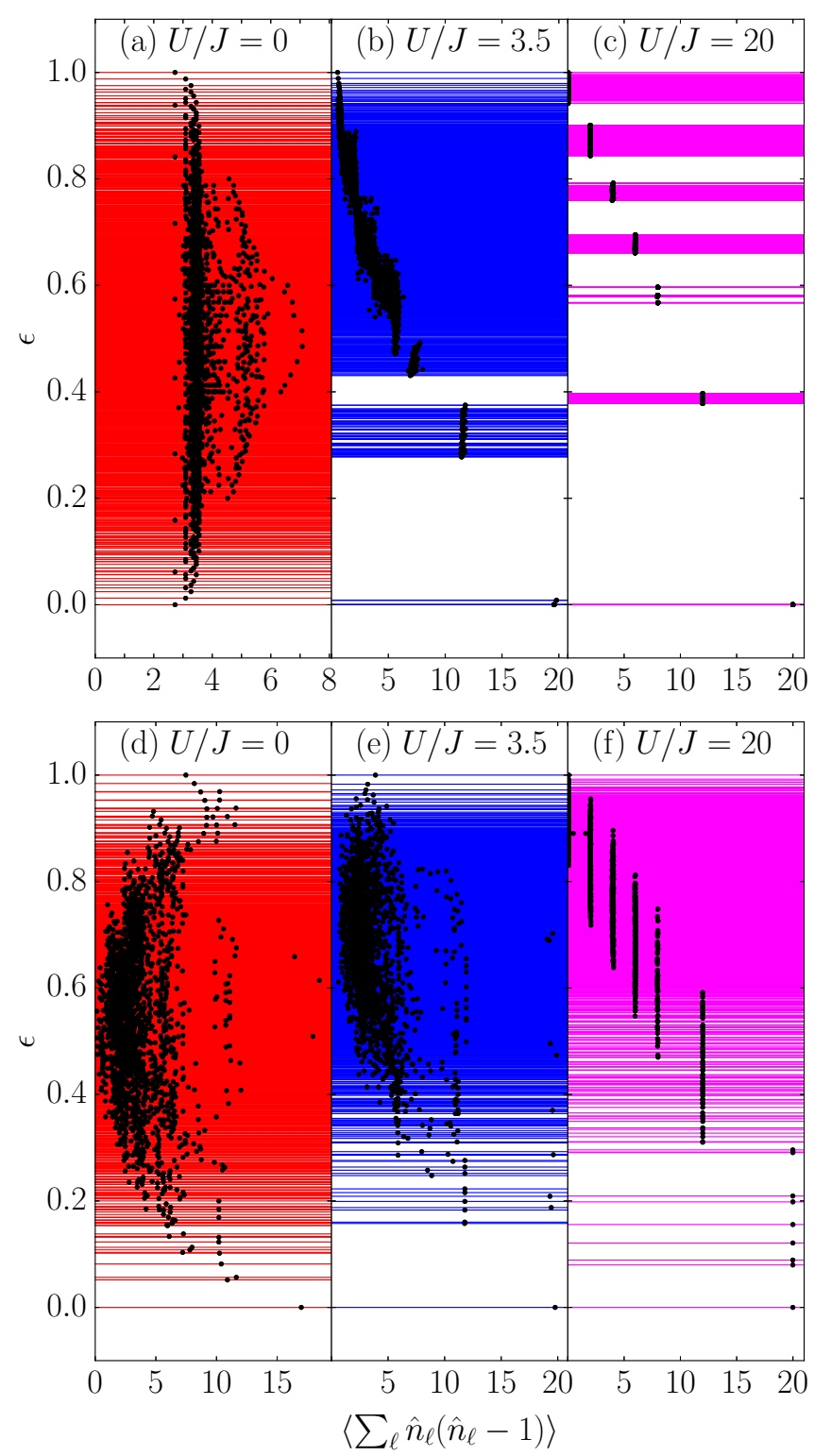

FIG. 2. Normalized eigenenergies of the clean (a)-(c) and the disordered (d)-(f) Bose-Hubbard Hamiltonian at half-filling and $L=$ 10 for anharmonicities $U / J=0$ (a) and (d); $U / J=3.5$ (b) and (e); $U / J=20$ (c) and (f). The eigenenergies are scaled as $\epsilon=$ $\left(E-E_{\min }\right) /\left(E_{\max }-E_{\min }\right)$. The disorder realization, drawn from a uniform distribution with the disorder strength $W / J=10$, is the same for each $U$. Black dots show the expectation value of the total anharmonicity operator $\sum_{\ell} \hat{n}_{\ell}\left(\hat{n}_{\ell}-1\right)$ in the corresponding eigenstate.

of the conservation of total number of quanta, Hamiltonian is a block-diagonal matrix where each block is characterized by a total number of excitations $\langle\hat{N}\rangle=N$. In a system with $L$ sites, these individual blocks have dimensions

$$
D_{N, L}=\frac{(N+L-1) !}{(L-1) ! N !} \approx \frac{\left[(1+f)\left(1+f^{-1}\right)^{f}\right]^{L}}{\sqrt{L}},
$$

where the last form is an approximation in the long-chain limit with filling factor $f=N / L$. For a spin chain the size of a sector with zero total magnetization (the largest sector) scales approximately as $2^{L} / \sqrt{L}$ [65]. In the long-chain limit, the Bose-Hubbard chain with half-filling scales roughly as $2.6^{L} / \sqrt{L}$, and with unit filling already as $4^{L} / \sqrt{L}$. This naturally implies that we cannot reach as large system sizes as used in spin chain studies, where the currently reached upper limit is $L=24$ [65] for exact numerical studies of the Hamiltonian eigenstates. The largest spin chain studies rely on massive parallelization on distributed memory machines. Our simulations use smaller computational resources, but we are still able to study systems with 14 transmons, which have Hilbert space roughly comparable to that of spin-1/2 chain with 18 sites. The chain with 16 transmons is already comparable to 22 spins, so it seems to be the current practical upper limit for this kind of study.

Technically, in a sector with $N$ quanta, each transmon needs $N+1$ lowest energy levels. One can then construct all the necessary operators in this desired sector. This can be done by first building all the possible Fock state configurations in this sector, i.e., states $\left|n_{1}, n_{2}, n_{3}, \ldots, n_{L}\right\rangle$ with $\sum_{\ell=1}^{L} n_{\ell}=N$. One can then obtain the necessary matrix elements by considering how the individual operators such as $\hat{a}_{1}^{\dagger} \hat{a}_{2}$ should operate on these basis states. The operators are most efficiently implemented as sparse matrices, which is done here with Eigenlibrary [66] for $\mathrm{C}++$. The resulting Hamiltonian is a sparse matrix. However, we note that the next-nearest-neighbor interaction of Hamiltonian (3) reduces the sparseness and makes exact diagonalization numerically more demanding problem than the regular Bose-Hubbard model. For more details of the exact diagonalization, see Appendix A.

\section{B. Flux-tunable disorder potential}

Since our goal is to study the many-body localization phase transition tuned by disorder, we explore the means to control disorder amplitude in situ. With transmons, this is possible via overall magnetic flux tuning [67]. A single transmon can be made to consist of two parallel Josephson junctions with energies $E_{\mathrm{J} 1}$ and $E_{\mathrm{J} 2}$ [2] connected by a loop with a surface area $A$; see Fig. 1. In this case, the on-site energy of a transmon depends on the magnetic flux $\Phi=B A$ induced by a uniform magnetic field $B$ threading the loop. Thus, the on-site energy of a transmon is [2]

$$
\omega(\Phi)=\frac{\sqrt{8 E_{\mathrm{C}} E_{\Sigma \mathrm{J}}}}{\hbar} \sqrt[4]{\cos ^{2}\left(\frac{\pi \Phi}{\Phi_{0}}\right)+d^{2} \sin ^{2}\left(\frac{\pi \Phi}{\Phi_{0}}\right)},
$$

where $\Phi_{0}=h / 2 e$ is the superconducting flux quantum, $E_{\Sigma \mathrm{J}}=$ $E_{\mathrm{J} 1}+E_{\mathrm{J} 2}$ is the sum of the junction energies and $d=$ $\frac{E_{11}-E_{12}}{E_{11}+E_{12}} \sim \pm 0.1$ is the junction asymmetry. We have neglected the constant term $-E_{\mathrm{C}} / \hbar$.

The process of multiple transmon fabrication typically results in a Gaussian distributed variation of the loop areas $A_{\ell}$ of the resulting devices. If an array constructed from these transmons is placed in the external magnetic field, a Gaussian distributed magnetic flux $\Phi_{\ell}=B A_{\ell}$ is induced in each transmon, which leads to nonuniformly distributed 


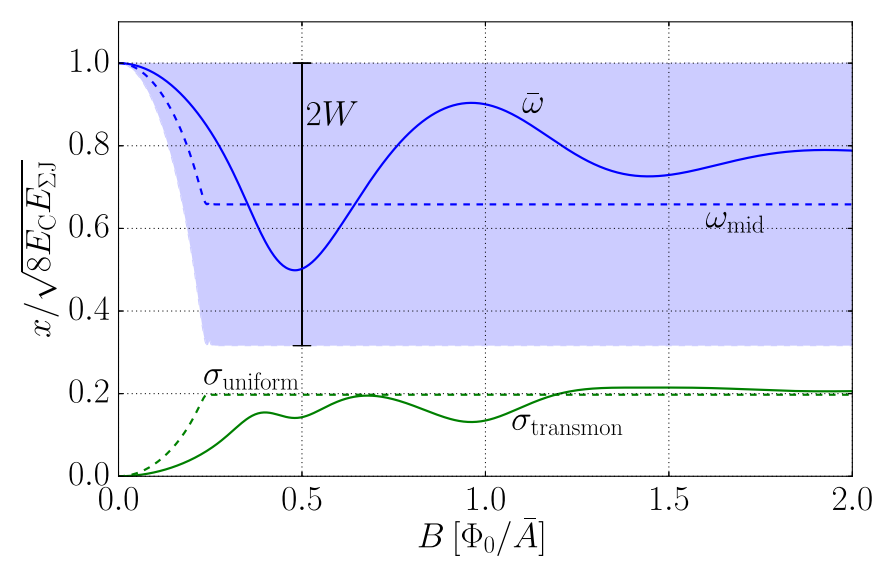

FIG. 3. Behavior of the mean energy $\bar{\omega}$ and corresponding standard deviation $\sigma_{\text {transmon }}$ of the transmon potential as a function of magnetic field. The fact that the mean energy (blue curve) is close to the "middle" energy $\omega_{\text {mid }}=\left(\omega_{\max }+\omega_{\min }\right) / 2$ (blue dashed curve) at high magnetic fields suggests that the uniform distribution provides a good approximation to the true distribution of $\omega_{l}$. The standard deviation (green curve) of the transmon disorder potential of Eq. (7) as a function of magnetic field also becomes close to the one of the uniform distribution $\sigma_{\text {uniform }}=W / \sqrt{3}$ (dashed green curve) for strong magnetic field. In contrast, weak magnetic fields give the narrow distribution of transmon energies sharply peaked around the mean energy. Junction asymmetry is $d=0.1$, loop areas are assumed to be Gaussian distributed with standard deviation $1 / 5$ of the mean $\bar{A}$, and all the values are expressed in the units of unperturbed transmon energy $\sqrt{8 E_{\mathrm{C}} E_{\Sigma \mathrm{J}}}$.

on-site energies $\omega_{\ell}$,

$$
\omega_{\ell}(B)=\frac{\sqrt{8 E_{\mathrm{C}} E_{\Sigma \mathrm{J}}}}{\hbar} \sqrt[4]{\cos ^{2}\left(\frac{\pi B A_{\ell}}{\Phi_{0}}\right)+d^{2} \sin ^{2}\left(\frac{\pi B A_{\ell}}{\Phi_{0}}\right)} .
$$

The energy is bounded above by $\omega_{\max }=\sqrt{8 E_{\mathrm{C}} E_{\Sigma \mathrm{J}}} / \hbar$ and the junction asymmetry sets the lower bound $\omega_{\min }=\sqrt{|d|} \omega_{\max }$. Since the energy depends nonlinearly on the magnetic flux, both the mean energy and the variance of the nonuniform energy distribution of Eq. (7) can be in situ controlled with the uniform magnetic field $B$, as visualized in Fig. 3 .

The resulting distribution of $\omega_{\ell}$ is nonuniform, so that in general the mean energy $\bar{\omega}$ differs from the average of largest and smallest energy. Nevertheless, the mean energy is the same for each transmon and has no effect on the many-body dynamics since it can be removed by switching to a rotating coordinate system with $\hat{U}=\exp \left(-i \bar{\omega} t \sum_{\ell} \hat{n}_{\ell}\right)$. The random energies are distributed around their mean value with width $2 W=\omega_{\max }-\omega_{\min }$; see Fig. 3 . Thus, we can use the parameter $W$ as an approximate disorder strength and assume that the distribution is uniform, $\omega_{\ell} \sim[-W, W]$ with the resulting standard deviation $W / \sqrt{3}$.

In Fig. 3, we show that the disorder strength $W$ exhibits nonlinear growth at weak magnetic field, but it quickly saturates due to the saturation of the minimum energy to value $\omega_{\min }=\sqrt{|d|} \sqrt{8 E_{\mathrm{C}} E_{\Sigma \mathrm{J}}}$. Notice that because loop area is correlated with neither the total Josephson energy nor the charging energy, in the absence of magnetic field and assuming no fabrication disorder in junctions, all the transmons are nominally identical. Weak fabrication disorder [34] breaks this by inducing a lower bound for the attainable experimental disorder $W_{\min } / 2 \pi \approx 10 \mathrm{MHz}$. In practice, however, the exact form for the disorder potential influences only nonuniversal details, such as the exact location of the phase transition. Therefore, in what follows, we use the uniform distribution instead of the transmon potential due to its simpler form and to facilitate the comparison with other studies of many-body localization [15-17,23,68].

\section{Disordered Bose-Hubbard model}

As discussed above, the on-site energy can be made strongly disordered by flux tuning. In addition, anharmonicity and tunneling terms can also contain disorder through fabrication and dependence on the flux-tunable Josephson energy. We can write the Hamiltonian of the disordered BoseHubbard model as

$$
\begin{aligned}
\hat{H} / \hbar= & \sum_{\ell=1}^{L} \omega_{\ell} \hat{n}_{\ell}-\sum_{\ell=1}^{L} \frac{U_{\ell}}{2} \hat{n}_{\ell}\left(\hat{n}_{\ell}-1\right) \\
& +\sum_{\ell=1}^{L-1} J_{\ell}\left(\hat{a}_{\ell}^{\dagger} \hat{a}_{\ell+1}+\hat{a}_{\ell} \hat{a}_{\ell+1}^{\dagger}\right),
\end{aligned}
$$

and we can also include disordered higher order anharmonicity and next-nearest-neighbor hopping in Eqs. (2) and (3). Experimentally achievable parameters for this Hamiltonian are listed in Table I. The disorder in hopping and on-site interaction can be drawn, e.g., from a Gaussian distribution. Because the disorder in the on-site energy reaches much larger values than is possible for the hopping and on-site interaction, we mainly focus on the situation where disorder is included only in the on-site energies $\omega_{\ell}$ of Eq. (8).

The many-body eigenenergies of the disordered BoseHubbard model are shown in Figs. 2(d)-2(f). We observe that the disorder changes the structure of the spectrum from that of the clean system shown in Figs. 2(a)-2(c). Because sites are no longer identical, there exists a preferred site with the lowest on-site energy and the configuration where all excitations occupy this site gives a good approximation to the ground state of the system. For sufficiently weak anharmonicity, the disorder dominates and the expectation value of the total anharmonicity operator can be large even in high-energy eigenstates. For strong anharmonicity, minibands of the clean system start to overlap, and the clean system structure, where the expectation value of the total anharmonicity operator decreases with increasing energy, still remains.

\section{Density of states}

The interplay of disorder and anharmonicity also has impact on the density of states, which varies between different disorder realizations, as shown in Fig. 4. Especially we note that the normalized energy at which the density of states has a maximum depends on the realization, the disorder strength $W$ and the on-site interaction $U$. The same behavior is also observed with the density of states plotted as a function of energy $E$ rather than rescaled energy $\epsilon$. 


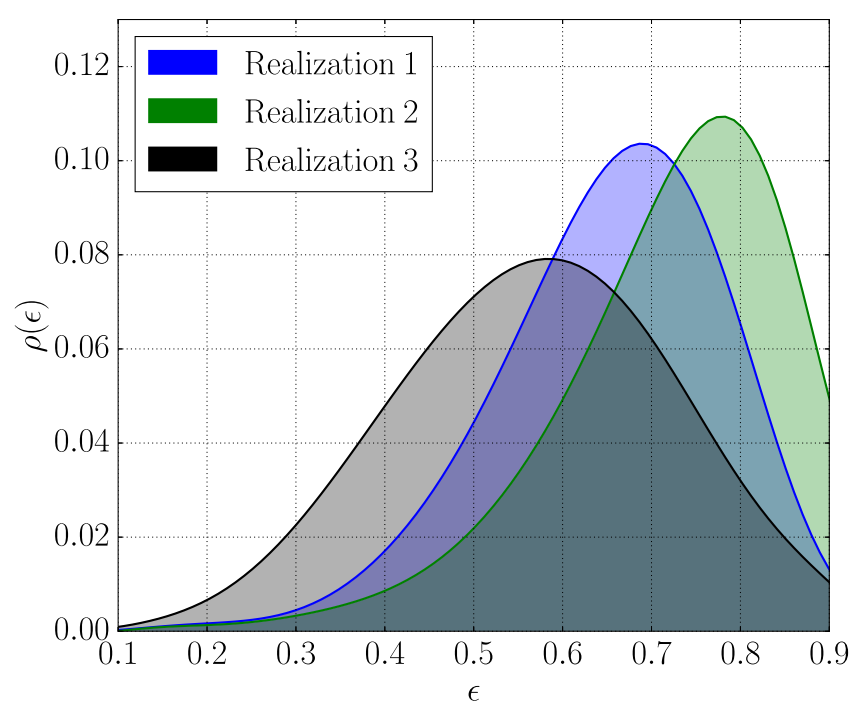

FIG. 4. The density of states $\rho(\epsilon)$ in the disordered BoseHubbard model of Eq. (8) as a function of normalized energy $\epsilon$ for three different disorder realizations with parameters $L=10$, $U / J=3.5$, and $W / J=10$. The location of the maximum varies due to interplay of anharmonicity and disorder. The realization 1 (blue) is the same as in Fig. 2(e).

Since many-body localization is a property of highly excited eigenstates, the disorder-dependent density of states introduces an additional complexity. In the most studied model of the many-body localization, the Heisenberg spin chain $[15,16]$, the spectrum is symmetric and the density of states has much weaker dependence on disorder realizations with a maximum located in the middle of the spectrum $(\epsilon=$ 0.5 ). Thus, in that model one can choose some fixed normalized energy and compare the corresponding many-body eigenstates and their properties (bipartite entanglement entropy, bipartite number uncertainty etc.) between different disorder realizations and eventually average the properties over a narrow energy window around the fixed normalized energy [15]. However, for the disordered Bose-Hubbard model with sufficiently large many-particle interaction strength $U$, this is not possible because the properties of eigenstates obtained this way would vary too much. For example, in one realization the many-body eigenstate closest to the fixed normalized energy $\epsilon=0.6$ might be close to the maximum density of states [see realization 3 (black) in Fig. 4] and thus exhibit infinite-temperature behavior, but in another [realization 2 (green) in Fig. 4] it might correspond to one of the low-lying eigenstates. Thus, in order to obtain comparable eigenstates, we instead choose them at the maximum density of states of each individual realization. This is a physically justified choice since in the Heisenberg spin chain the many-body localization transition is known to require the largest disorder strength in eigenstates located at the maximum density of states, due to the influence of the neighboring states, which gives rise to the many-body mobility edge [15]. Similar behavior is expected also in the disordered Bose-Hubbard model of Eq. (8). A downside is that we need at least an estimate for the density of states, which introduces additional numerical complexity.
An estimate of the density of states, without solving the full spectrum of many-body energy levels, can be obtained by using Sylvester's law of inertia [39], which requires computation of $L D L$ matrix decompositions of the Hamiltonian of Eq. (8). This results in an exact number of energy eigenvalues within a specified energy interval. If the interval size is sufficiently large, this method produces the density of states more efficiently than the full exact eigendecomposition. Since we are not interested in the full shape of the density of states but just the location of the maximum, the $L D L$-decompositionbased method is very efficient. For large systems, however, the $L D L$ decomposition [essentially scaling similarly as eigendecomposition, $\mathcal{O}\left(n^{3}\right)$, where $n$ is the matrix dimension, but with a smaller prefactor] becomes numerically too heavy. Luckily, accurate and efficient approximation of the number of eigenvalues within a specified energy interval, and hence the density of states, can still be obtained with the stochastic Chebyshev expansion method [39]. This method is efficient for large systems for two main reasons. First, as a stochastic method, accuracy can be traded to speed similar to Monte Carlo methods. Second, important for large systems, this method is based only on sparse matrix-vector multiplications instead of matrix decomposition. The details of Sylvester's law of inertia, $L D L$ decomposition, and the stochastic Chebyshev expansion method are presented in Appendix B.

\section{PHASE TRANSITION}

Generic, isolated, and interacting many-body quantum systems reach thermal equilibrium in course of their unitary dynamics [8]. The eigenstate thermalization hypothesis $(\mathrm{ETH})$ provides a microscopic mechanism for thermalization, by imposing the condition that individual eigenstates of the many-body system have thermal expectation values of all local observables $[69,70]$. Eigenstate thermalization hypothesis has numerous implications for the structure of eigenstates. In particular, it suggests a volume-law entanglement in highly excited eigenstates.

However, the sufficiently large disorder may lead to the many-body localized phase that is characterized by the breakdown of thermalization $[4,6]$. The many-body localized phase can be viewed as the interacting cousin of Anderson insulator [71]. However, the presence of interactions leads to qualitatively different properties, in particular allowing longdistance entanglement spreading in the many-body localized phase. In addition, by increasing interactions and/or decreasing disorder, one can tune the transition between many-body localized and thermalizing phases [17].

The many-body localization phase transition is a dynamical phase transition that occurs in highly excited eigenstates. This transition separates the thermalizing phase where eigenstates are obeying eigenstate thermalization hypothesis from many-body localized phase characterized by emergent local integrals of motion $[4,6]$. Consequently, one can diagnose the many-body localization phase transition by observing the breakdown of eigenstate thermalization hypothesis in highly excited eigenstates manifested in the scaling of the bipartite entanglement entropy, the bipartite fluctuations of global conserved quantities, participation ratios of many-body eigenstates, and distribution of adjacent energy level spacings [17]. 
In this section, we characterize the critical disorder strength $W_{\mathrm{c}}$ as a function of interaction strength $U$ at which the phase transition between the ergodic and many-body localized phase occurs in the disordered Bose-Hubbard model (8). First, we introduce the different quantities used to diagnose the phase transition. Afterward, we present the phase diagram, discuss the influence of filling factor, and compare our results to earlier studies.

\section{A. Bipartite entanglement and number uncertainty}

The bipartite entanglement entropy between two parts of the system provides a natural quantity to diagnose the transition from ergodic to many-body localized phase. For an arbitrary state $|\alpha\rangle$, one can construct the corresponding density operator $\hat{\rho}=|\alpha\rangle\langle\alpha|$. We divide the system into two, left and right, partitions. The density operator for the left part of the chain $(A)$ is then obtained by tracing out the degrees of freedom of the right part $(B)$ from the full density operator,

$$
\hat{\rho}_{A}=\operatorname{Tr}_{B}(|\alpha\rangle\langle\alpha|) .
$$

The entanglement between the subsystems $A$ and $B$ is given by the von Neumann entropy of the subsystem density operator, defined as

$$
S=-\operatorname{Tr}_{A}\left(\hat{\rho}_{A} \ln \hat{\rho}_{A}\right),
$$

and it can be calculated efficiently with Schmidt decomposition [72]. In the ergodic phase, the entanglement entropy of a typical Hamiltonian eigenstate grows with the size of the subsystem $A-\mathrm{a}$ volume law scaling. In the localized phase, however, the entanglement scales according to an area law since eigenstates can be obtained by a quasilocal unitary acting on a product state $[4,6]$. The transition between these two distinct behaviors provides a tool for diagnosing the phase transition $[15,16]$.

Even though the Hamiltonian (8) conserves the total number of excitations, the number of excitations within a given half of the system, determined by the operator

$$
\hat{N}_{A}=\sum_{\ell=1}^{L / 2} \hat{n}_{\ell},
$$

is not fixed. The fluctuations of $\hat{N}_{A}$ can be used as a characteristic measure between delocalization and localization. The particle number uncertainty is defined through the variance of the half-system particle number operator $\hat{N}_{A}$,

$$
F=\left\langle\alpha\left|\hat{N}_{A}^{2}\right| \alpha\right\rangle-\left\langle\alpha\left|\hat{N}_{A}\right| \alpha\right\rangle^{2},
$$

and it shows similar behavior as the entanglement entropy in ergodic and localized phases [15,16,73].

We have studied how the disorder strength $W$ and the on-site interaction $U$ affect the bipartite entanglement entropy $S$ and the bipartite number uncertainty $F$ in different sized systems for equal bipartition of the transmon chain: Data for $U / J=3.5$ value is shown in Fig. 5. The eigenstate $|\alpha\rangle$ for which these properties are calculated is selected to be the one closest to the estimated maximum of the density of states for each disorder realization. We observe that with weak disorder both observables scale according to the volume law, but as the disorder increases the scaling turns to the area

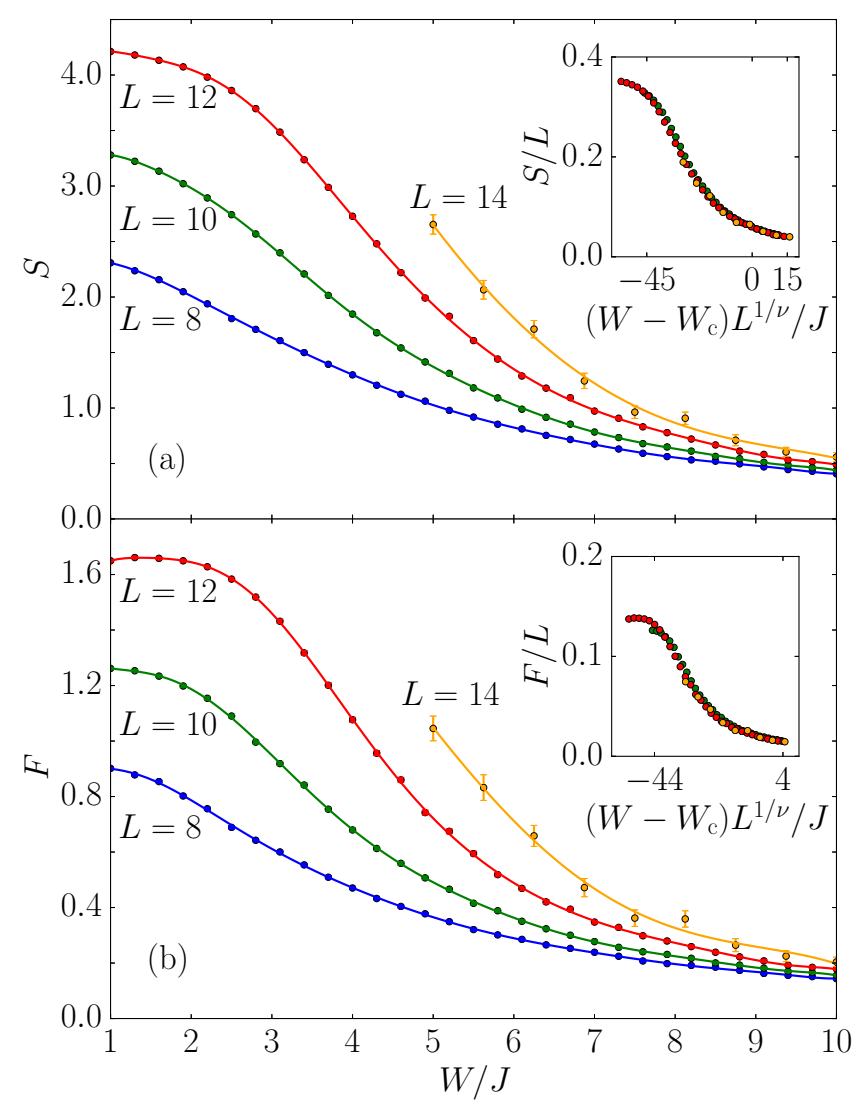

FIG. 5. The bipartite entanglement entropy $S$ (a) and the bipartite number uncertainty $F($ b) as a function of the disorder strength $W$ of uniform disorder distribution for different system sizes $L=$ 8 (blue), 10 (green), 12 (red), and 14 (yellow) with $U / J=3.5$. The eigenstate, for which the properties are calculated, is the one closest to the maximum density of states. The results are averaged over 4000 disorder realizations, except in $L=14$ we have 264 realizations. Dots denote our data points, and the curves are polynomial fits for visibility. The standard error is denoted with the error bars, and for shorter chains they are smaller than the marker size. In the insets, we present the collapsed data from a finite-size scaling analysis with the ansatz $g\left[L^{1 / v}\left(W-W_{\mathrm{c}}\right)\right]$ without the shortest chain $L=8$. With this value of $U$, we obtain critical disorder strength $W_{c} / J=8.24$ and scaling exponent $v=1.126$ for the bipartite entanglement entropy and for the bipartite number uncertainty $W_{c} / J=9.36$ and $v=1.24$.

law, which signals the presence of a phase transition from ergodic to localized phase. For large on-site interaction $U$, the eigenstates form the miniband structure, as discussed in Sec. II. In such a situation, the density of states has several local maxima, and the selection of the eigenstates is no longer clear. However, the miniband structure rapidly vanishes with increasing $W$ for the studied values of $U$, and therefore it does not affect the results.

\section{B. Energy level statistics}

The bipartite entanglement entropy and bipartite number uncertainty are properties of the Hamiltonian eigenstates, but the distinction between ergodic and localized phases is also visible in the distribution of the energy eigenvalues. A widely used tool for measuring it is the energy level 


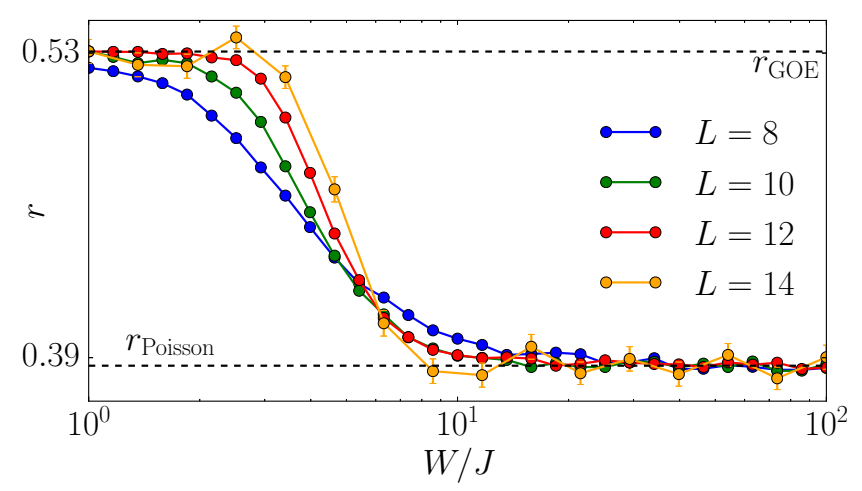

FIG. 6. Average adjacent gap ratio $r$ as a function of the disorder strength $W$ for different sized systems with anharmonicity $U / J=$ 3.5. The reference values for Gaussian orthogonal ensemble $\left(r_{\mathrm{GOE}}\right)$ and Poissonian distribution $\left(r_{\text {Poisson }}\right)$ are denoted by black horizontal lines. The results are averaged over 4000 realizations, except for $L=14$, which is averaged over 264 realizations. The eigenvalues are located at the maximum density of states, and the gap ratio is calculated over 16 adjacent eigenstates from each disorder realization. The error bars denote the standard error and are in general smaller than the marker size.

spacing distribution. In the ergodic phase, the eigenvalues are distributed according to the Gaussian orthogonal ensemble, while in the localized phase they are uncorrelated and obey Poissonian statistics [8]. One often considers the adjacent gap ratio $[12,15,17,68]$

$$
r^{(n)}=\frac{\min \left[\delta^{(n)}, \delta^{(n+1)}\right]}{\max \left[\delta^{(n)}, \delta^{(n+1)}\right]},
$$

where $\delta^{(n)}=E_{n}-E_{n-1}>0$ is the energy difference between a pair of adjacent levels. In the ergodic phase, the average adjacent gap ratio over $n$ is $r_{\mathrm{GOE}} \approx 0.536$, and in the localized phase $r_{\text {Poisson }} \approx 0.386$ [74]. We have also studied the distribution of the adjacent gap ratios as a function of disorder strength around the maximum of the density of states. At weak disorder, the average gap ratio is consistent with the Gaussian orthogonal ensemble [74], whereas for strong disorder energy levels become essentially uncorrelated and the average gap ratio tends to $r_{\text {Poisson }}$, as shown in Fig. 6. An estimate for the transition point can be obtained from the point at which the lines of different sized systems cross. However, in order to obtain convergent results for the transition point, one has to calculate a large number of eigenvalues from each realization, which makes the adjacent gap ratio computationally much more expensive than the bipartite entanglement and number uncertainty for longer chains. For this reason, we have not used the average adjacent gap ratio in further studies of the phase transition.

\section{Phase diagram}

An estimate for the critical disorder strength $W_{\mathrm{c}}$ can be obtained from the data in Fig. 5 by scaling the curves with corresponding chain lengths and determining the disorder strength at which the curves cross. Another possibility, the one that we use, is the finite-size scaling collapse using ansatz $g\left[L^{1 / v}\left(W-W_{\mathrm{c}}\right)\right]$, which collapses the data to a single

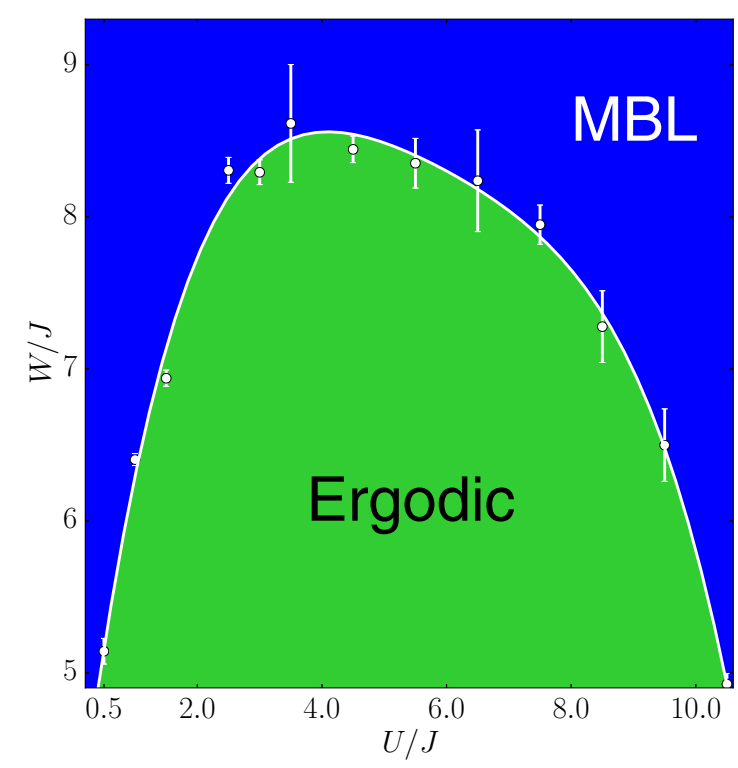

FIG. 7. Phase diagram for the half-filled disordered BoseHubbard Hamiltonian (8) as a function of the on-site interaction $U$ and the disorder strength $W$. The transition point is estimated using finite-size scaling analysis for different sized systems $L=10,12,14$ for the bipartite entanglement entropy and the bipartite number uncertainty, shown in Fig. 5. The disorder is included only in the onsite energy $\omega_{\ell}$ and it is drawn from the uniform distribution $[-W, W]$. The data points are obtained as an average of the transition points given by the finite-size scaling analysis on the bipartite entanglement entropy and the bipartite number uncertainty and the error bars show the deviation between these two values. The white curve is a polynomial fit that improves visual clarity.

curve [15,75] (see insets of Fig. 5). We obtain the critical disorder strength as a function of the anharmonicity $U$ for both the bipartite entanglement entropy and the bipartite number uncertainty and use their average as an estimation for the transition point. The phase diagram shown in Fig. 7 is constructed from this data. The overall shape of the phase diagram is similar to the corresponding fermionic system studied in Ref. [68]. However, the maximum critical disorder strength is reached at much weaker on-site interactions in the bosonic system compared to the fermionic case. We attribute this behavior to the fact that the on-site interaction is effectively much stronger in the bosonic case since the number of excitations per site is unlimited.

From the point of view of experimentally realizable superconducting transmon circuits, the attainable parameter regime (see Table I) is roughly $U / J=2-30$ and $W / J=0.1-200$, yielding that the phase transition occurs within the experimentally realizable parameter range. Furthermore, based on our additional calculations (not shown here) with experimentally relevant parameters, the additional perturbations by the higher order anharmonicity in Eq. (2) and the next-nearest-neighbor hopping in Eq. (3) do not change the situation and the phase diagram remains intact within the original error bars. However, if the next-nearest-neighbor hopping $J_{2}$ is made strong enough, $J_{2} \gtrsim J / 5$, the transition point shifts toward larger disorder strengths. This can be qualitatively understood as an increase in kinetic energy, promoting delocalization [24]. 
The higher order on-site interaction $U_{2}$, on the other hand, effectively weakens the anharmonicity due to opposite sign. With sufficiently large values $U_{2} \gtrsim U / 3$, the higher order anharmonicity shifts the transition points toward larger $U$. Finally, in systems where the disorder is included also in the hopping and on-site interaction terms, the phase transition occurs at slightly weaker disorder strengths since the system is more disordered.

We have also studied the phase transition also for systems with the transmon disorder of Eq. (7) and observed that the critical disorder strength corresponds to a somewhat larger $(\lesssim J)$ disorder strength than with the uniform disorder. This happens because disorder distributions with larger standard deviations are more effective at localizing the system. Indeed, in the studied disorder strength range, the uniform distribution has larger standard deviation than the transmon disorder (magnetic field values between 0.01 and 0.3 in Fig. 3). With large anharmonicities, the on-site interaction starts to dominate, and the critical disorder strength is within error bars the same for both disorder distributions.

The increase of the filling factor also increases the critical disorder strength. We attribute this to the bosonic enhancement of tunneling for multiply occupied sites, which makes the system more robust against localization. We have performed simulations for small systems $(L<10)$ with unit filling and confirmed this behavior. The numerical simulations with larger fillings are limited to smaller system sizes than in the half-filled case due to larger total Hilbert spaces, given by Eq. (5). Because there is no upper limit for the total number of excitations in bosonic systems, an open question remains regarding how critical disorder strength behaves at much larger fillings.

The phase transition was studied experimentally in Ref. [27], where a chain of nine transmons with two excitations in total was studied through adjacent energy gap and participation ratios. They used quasiperiodic potential $\omega_{\ell}=\Delta \cos (2 \pi \beta \ell)$, where $\Delta$ is the disorder strength and $\beta$ is an irrational number. Notice that the quasiperiodic potential with the disorder strength $\Delta$ has standard deviation $\Delta / \sqrt{2}$. Thus, when using standard deviation as a disorder measure the quasiperiodic potential is more random than the uniform disorder with disorder amplitude $W=\Delta$ and standard deviation $\Delta / \sqrt{3}$. For $U / J=3.5$, Roushan et al. in Ref. [27] found a phase transition at $\Delta / J \approx 2$, which corresponds to $W / J \approx 2.5$ for the uniform potential when the mapping is done through equal standard deviations between the two distributions. Such a small disorder strength compared to our phase diagram is explained partly by the small filling factor and partly by the more disordered potential.

\section{DYNAMICS}

All the eigenstate measures of localization that we have presented so far (eigenstate bipartite entanglement entropy, bipartite number uncertainty, and average adjacent gap ratio in Figs. 5 and 6) are shared between the phases of Anderson localization and many-body localization. The distinction between the two phases of localization as well as between the localized and ergodic phases can be observed in several dynamical properties, some of which are available also for experiments on superconducting circuits. In this paper, we consider only unitary dynamics. We utilize exact eigendecomposition only for the shortest system $L=8$, and for the larger systems $L=10,12,14$ the unitary time evolution is calculated through Krylov subspace methods; see Appendix C for details. Furthermore, we simulate the time evolution for a long chain of $L=40$ transmons to confirm that the results are properly saturated in the system size and exhibit no boundary effects. The long chain simulations are performed using timeevolving block decimation scheme for matrix product states, detailed in Appendix D.

Dissipation and decoherence are always present in superconducting circuits, and they are expected to eventually destroy the many-body localized phase [76-78]. However, due to long relaxation and coherence times of modern transmons ( $T_{1} \approx 60 \mu \mathrm{s}$ and $T_{2} \approx 20 \mu \mathrm{s}$ ) [40], the system dynamics remain unitary to relatively long times, about $10 \mu \mathrm{s}$. Based on our simulations, this is sufficient for observing the distinct behaviors of all three phases.

\section{A. Dynamics of bipartite entanglement entropy}

Let us start by considering the quench dynamics under the disordered Bose-Hubbard Hamiltonian of Eq. (8). We perform the time evolution of the nonentangled initial state $\left|\psi_{0}\right\rangle$ and measure the dynamics of the entanglement entropy $S(t)$. Our choice for the initial state is a Néel-type of state $|101010 \ldots\rangle$ studied also in experimental setups [41]. The dynamics of the bipartite entanglement entropy $S(t)$ for different sized systems is shown in Figs. 8(a) and 8(d). Our main interest is in the differences of the dynamical behavior in the many-body localized, Anderson localized, and ergodic phases.

At strong disorder in Fig. 8(a), the early dynamics of the bipartite entanglement entropy $S(t)$ for the interacting (many-body localized) and noninteracting (Anderson localized) systems are similar, that is, they both initially exhibit similar rapid growth of entanglement. However, for the interacting system, the information spreading does not stop after reaching the length scales of the localization length but instead continues at much smaller rate than initially. This logarithmic growth of entanglement at long times is caused by the interaction-induced dephasing $[7,16,18,19,79]$ present only in the many-body localized phase. The interacting system eventually equilibrates, but not to the canonical ensemble $[5,80]$. Thus, in the many-body localized phase there exists two distinct regions in time evolution. During the first one, the excitation quanta explore the surrounding region within the localization length. After this, they start to slowly dephase with other particles further apart, which leads to the described long-time behavior.

At weak disorder in Fig. 8(d), information rapidly spreads and eventually reaches thermal equilibrium as expected in the ergodic phase [8]. The entanglement entropy $S(t)$ has a ballistic growth and saturates to a value that obeys the volume law [81] [inset of Fig. 8(d)]. After the saturation and thermal equilibration, local observables are determined by canonical ensemble [5,36]. In the Anderson localized phase at $U=0$ [dashed line in Fig. 8(a)], the dynamics is constrained within the localization length and the entanglement entropy saturates to a much smaller value than in the ergodic phase. This 

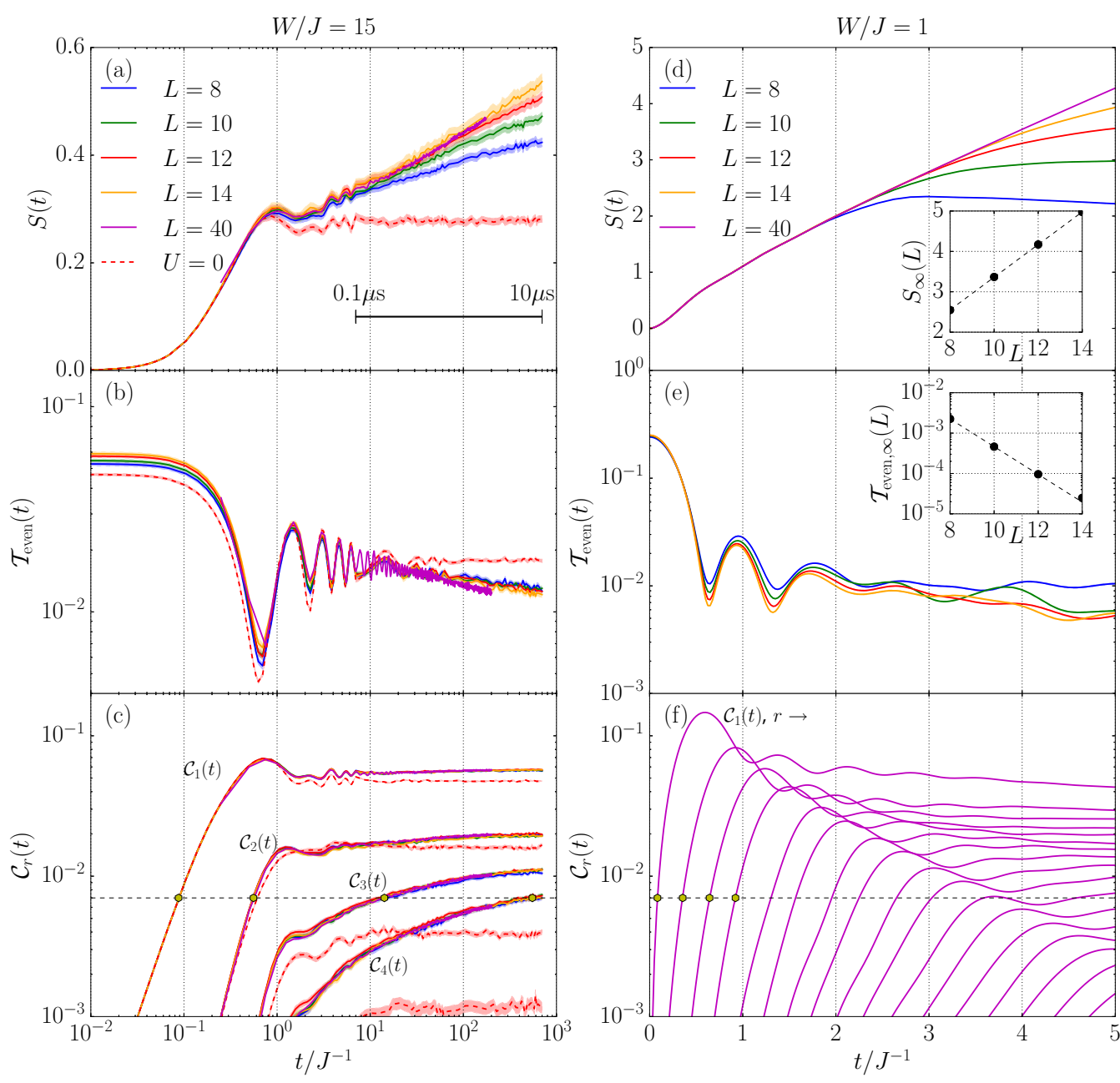

FIG. 8. The bipartite entanglement entropy $S(t)\left[(\mathrm{a})\right.$ and (d)], the temporal number fluctuation $\mathcal{T}_{\text {even }}(t)$ of even sites [(b) and (e)], and the two-site number correlations $\mathcal{C}_{r}(t)[(\mathrm{c})$ and (f)] as a function of time $t$. The dynamical probes are calculated for interacting system with $U / J=3.5$ (solid lines) for system sizes $L=8$ (blue), 10 (green), 12 (red), 14 (yellow), and 40 (purple), and for the noninteraction situation $U / J=0$ with $L=12$ sites (dashed red line). The disorder distribution is uniform, and panels (a)-(c) correspond to strong disorder $W / J=15$ and panels (d)-(f) to weak disorder $W / J=1$. Results for system sizes $L \leqslant 12$ are averaged over 2000, for $L=14$ over 1000 , and for $L=40$ over 500 disorder realizations. For $L=40$, additional truncation of on-site Hilbert space was applied with maximal occupancy limited to $n_{\max }=4$ for $W / J=1$ and $n_{\max }=3$ for $W / J=15$, which is justified by the dilute initial state and rather short evolution times. Shaded regions depict the standard errors of the disorder averages. In panels (c) and (f), the yellow dots depict the points at which the corresponding correlations reach the predetermined reference value (dashed horizontal line). The insets in panels (d) and (e) describe the equilibrium values of the corresponding observables as a function of system size. Note also the different time scales in left and right columns.

saturation value depends on the localization length and is independent on the system size (not shown here). Anderson localized systems never reach thermal equilibrium within the whole system [80].

\section{B. Dynamics of on-site number fluctuations}

The entanglement entropy provides a good measure for identifying the many-body localized phase, and recent experiments with optical lattices [33] and trapped ions [35] have demonstrated that it can also be measured without mapping the full density matrix. We expect that similar schemes can be extended also to superconducting circuits. However, it is still beneficial to study simple observables that require measurements of only a few local expectation values and show the distinction between the three phases. Such observables are more accessible in experiments. One possibility is to study temporal fluctuations of local observables $[82,83]$. We consider here dynamics of fluctuations for the number operator $\hat{n}_{\ell}$ of the site $\ell$, defined as

$$
\mathcal{T}_{\ell}(t)=\left\langle\left[\left\langle\hat{n}_{\ell}(t)\right\rangle-\bar{n}_{\ell}\right]^{2}\right\rangle_{\mathrm{d}},
$$

where $\langle\cdot\rangle_{\mathrm{d}}$ denotes average over disorder realizations. The fluctuations are measured with respect to a steady-state, equilibrium value defined here as a long-time average

$$
\bar{n}_{\ell}=\lim _{t \rightarrow \infty} \frac{1}{t} \int_{0}^{t}\left\langle\hat{n}_{\ell}(\tau)\right\rangle d \tau .
$$

For additional convergence, we have averaged the temporal fluctuations over even and odd sites separately, and denoted these averages as $\mathcal{T}_{\text {even }}(t)$ and $\mathcal{T}_{\text {odd }}(t)$, but the results are similar also for individual sites. Fluctuations averaged over 
even sites are shown at strong disorder in Fig. 8(b) and at weak disorder in Fig. 8(e). At weak disorder, only results from the exact diagonalization are shown, since the time-evolving block decimation is restricted to short times and thus the long-time average is not accessible. Experimental advantage of this measure is that it requires only measurement of a single site or few sites and it can be achieved in high precision with superconducting circuits through dispersive readout that naturally measures transmon occupation $\left\langle\hat{n}_{\ell}\right\rangle$ [84].

Monitoring temporal fluctuations of a local operator can be seen as a measure for attainable volume that an initial excitation can explore. In Anderson localized systems, temporal fluctuations never vanish [83] since the effective volume is strictly limited by the localization length, seen in Fig. 8(b) (dashed line). In the many-body localized phase, after transients, the fluctuations decay as the power law [Fig. 8(b)]

$$
\mathcal{T}_{\ell}(t) \propto t^{-b},
$$

being a signal that after the initial buildup of the wave function the effective volume is slowly expanding $[82,83]$. Curiously, there is a clear distinction at later times between the powerlaw-like decay in the many-body localized phase and the saturation in the Anderson localized phase, but this distinction becomes visible roughly one order of magnitude later than in the bipartite entanglement entropy. In the thermal phase [Fig. 8(e)], we observe a rapid decay of the fluctuations until saturation to a value that depends on the system size as $e^{-a L}$, where $a$ is some positive constant [inset of Fig. 8(e)].

\section{Dynamics of two-site correlations}

Finally, we study the propagation of information using two-site number correlations [80,83]. For two sites separated by distance $r$, the correlations are defined as

$$
\mathcal{C}_{\ell, r}(t)=\left\langle\left|\left\langle\hat{n}_{\ell}(t) \hat{n}_{\ell+r}(t)\right\rangle-\left\langle\hat{n}_{\ell}(t)\right\rangle\left\langle\hat{n}_{\ell+r}(t)\right\rangle\right|\right\rangle_{\mathrm{d}} .
$$

In order to improve convergence, we average over each pair with fixed separation $r$ to obtain the distance-dependent correlation $\mathcal{C}_{r}(t)$,

$$
\mathcal{C}_{r}(t)=\frac{1}{L-r} \sum_{\ell} \mathcal{C}_{\ell, r}(t)
$$

where $L-r$ is the number of $r$ separated pairs in chain of $L$ sites. The conclusions are similar also for individual pairs. Experimentally, this might be more challenging to measure than the temporal fluctuations due to the limited ability to access the two-site correlation functions.

The time evolution for the four nearest correlations $\mathcal{C}_{1}(t)-$ $\mathcal{C}_{4}(t)$ is shown in Fig. 8(c) for strong disorder for different system sizes. In Fig. 8(f), we display the weak disorder case for $L=40$ and additional longer range correlations. In both figures, we have also displayed a reference value (dashed horizontal line) for monitoring the development of nearest correlations $\mathcal{C}_{1}(t)-\mathcal{C}_{4}(t)$ in different phases. In the thermal phase, the correlations first develop between adjacent sites and spread to more distant pairs at constant velocity; i.e., the peaks of the correlations are linearly spaced in time. Similarly, the correlations reach the reference value at equidistant times. The height of the peaks decays exponentially with the distance $r$, which implies exploration of finite fraction of Hilbert space up to Lieb-Robinson bounds [85]. After rapid initial dynamics, the correlations saturate to values inversely proportional to the system volume. The equilibrium values are all of the same magnitude.

In the localized systems, the correlations first increase rapidly. After the localization length is reached, the correlations in the Anderson localized system saturate to a value inversely proportional to the separation of the pairs. Saturated values are independent on the system size (not shown), and there is a difference of several orders of magnitude between different distances. In the many-body localized system, the correlations instead continue to grow at much smaller rates than initially. Because of this slow growth, the reference value for correlations at different distances is reached at logarithmically spaced times, revealing a logarithmic light cone. On the other hand, in the Anderson localized systems, the correlations never reach the reference value due to the saturation.

In summary, we conclude that the interacting and strongly disordered system resulting many-body localization is clearly distinguishable from that of noninteracting and ergodic phases by the presented dynamical probes shown in Fig. 8. Importantly, this dynamical distinction between the phases occurs at experimentally feasible timescales $0.1-10 \mu$ s [horizontal bar in Fig. 8(a)] set by the decoherence and dissipation rates of the modern superconducting ciruits.

\section{CONCLUSIONS}

In this work, we have numerically studied the many-body localization phase transition in the attractive Bose-Hubbard Hamiltonian using the methods of exact diagonalization as well as matrix product state dynamics. Such systems can be experimentally realized with arrays of superconducting circuits, and our purpose was to produce results that could be verified experimentally with currently available technology. The distinct features of many-body localization are visible in systems with a minimum of eight transmons, as shown in Fig. 8, although similar signatures can be observed already with six sites.

The bipartite entanglement entropy, bipartite number uncertainty, and adjacent gap ratio of the Hamiltonian eigenpairs exhibit ergodic behavior at weak and localized behavior at strong disorder. Using finite-size scaling analysis, we have obtained an estimate for the critical disorder strength as a function of the transmon anharmonicity and constructed the ergodic-many-body localized phase diagram for the attractive Bose-Hubbard Hamiltonian (Fig. 7). The phase transition occurs at experimentally feasible parameters and it is robust against higher order on-site interactions and longer range tunneling. The eigenstates were taken at the maximum density of states, which, due to the anharmonicity and bosonic nature of the system, is heavily dependent on the Hamiltonian parameters as well as on the disorder realization. Thus, in order to study comparable eigenstates, we had to estimate the density of states for each realization. For smaller systems, this was done with the $L D L$ matrix decomposition method that resorts to Sylvester's law of inertia. For larger systems, an approximation with the stochastic Chebyshev expansion was used. 
Distinction between the many-body localization and Anderson localization of noninteracting systems can be observed in the dynamics. We have simulated unitary quench dynamics and studied the time evolution of entanglement entropy, temporal number fluctuations, and two-site number operator correlations. These observables feature distinct behavior in ergodic, many-body localized, and Anderson localized phases. In the many-body localized phase, the entanglement displays logarithmic growth at long times, the temporal fluctuations decay according to a power law and the correlations spread logarithmically to more distant sites. This behavior becomes visible at experimentally relevant timescales, and thus we suggest that the temporal fluctuations and correlation functions are suitable dynamical observables for the experimental studies of the many-body localization in systems of superconducting circuits. We believe that the results presented in this paper will increase the attention and lead to focused experimental studies of many-body localization in systems of superconducting circuits.

The localization phenomenon ideally occurs in closed systems but it is known to survive for intermediate times in weakly open systems [78,86,87]. Dissipation and decoherence will have primarily different roles in the manybody localization of transmon arrays. Dissipation removes energy and excitations from the system, eventually bringing it into a dilute noninteracting phase, whereas decoherence destroys localization by destroying the phase coherence. The models for dissipation and decoherence of superconducting transmons are well known and characterized [50], which make it an excellent basis for studying open quantum system effects on many-body localization both experimentally and theoretically. Specific research questions are, for example, at what timescales will localization survive under dissipation and decoherence, and how these times depend on the filling factor, interaction, and disorder strengths and how continuous monitoring will affect localization through measurement back action. We leave addressing these questions for a future work.

\section{ACKNOWLEDGMENTS}

Special thanks are addressed to R. T. Brierley and S. M. Girvin for discussions and contributions in the early phase of the project. We are also grateful for fruitful discussion with Bryan K. Clark, Claudia De Grandi, Gerhard Kirchmair, Zaki Leghtas, Olli Mansikkamäki, Stefan Oleschko, Iivari Pietikäinen, Erkki Thuneberg, Sasu Tuohino, Jani Tuorila, and Xiongjie Yu. This research was financially supported by the Alfred Kordelin Foundation, the Emil Aaltonen Foundation, and the Academy of Finland under Grants No. 316619 and No. 320086. We also wish to acknowledge the CSC-IT Center for Science, Finland, and Finnish Grid and Cloud Infrastructure (persistent identifier urn:nbn:fi:research-infras2016072533) for computational resources.

\section{APPENDIX A: EXACT DIAGONALIZATION}

We are interested in the properties of the eigenstates and eigenvalues of the Hamiltonians of Eqs. (1) and (8). Because of the large dimensions and the need for several disorder realizations, the efficient full diagonalization is limited to small systems $L \leqslant 10$. However, we do not need the full spectrum, but only eigenpairs close to some specified target energy. The target-specified eigenpair can be obtained efficiently with the shift-and-invert method, where one considers an eigenvalue problem

$$
\mathrm{Hu}=\lambda \mathbf{u},
$$

where the eigenvector $\mathbf{u}$ is such that the eigenvalue is close to the target, $\lambda \approx \sigma$. To obtain this eigenpair efficiently, we can make a spectral shift and consider the matrix $(\mathrm{H}-\sigma \mathrm{l})^{-1}$. The largest eigenvalue of the matrix $(\mathrm{H}-\sigma \mathrm{l})^{-1}$ corresponds to the eigenvalue of the matrix $\mathrm{H}$ closest to the target $\sigma$.

Thus, we obtain a new eigenvalue problem,

$$
(H-\sigma \mathrm{I})^{-1} \mathbf{u}=\frac{1}{\lambda-\sigma} \mathbf{u} .
$$

The eigenvector $\mathbf{u}$ can then be obtained with the power iteration method where one repeatedly applies the matrix $(\mathrm{H}-\sigma \mathrm{I})^{-1}$ to an initial random vector $\mathbf{u}_{0}$ and normalizes the result:

$$
\mathbf{u}_{k+1}=\frac{(\mathrm{H}-\sigma \mathrm{l})^{-1} \mathbf{u}_{k}}{\left\|(\mathrm{H}-\sigma \mathrm{l})^{-1} \mathbf{u}_{k}\right\|} .
$$

After a suitable number of iterations, one obtains the eigenvector of the original matrix with the eigenvalue closest to the target. Because inverting a large matrix is a challenging operation, it is customary to convert the inverted matrix-vector multiplication to a system of linear equations subsequently solved with the $L U$ decomposition $[15,36,65]$, where $L$ and $U$ are lower and upper triangular matrices. We have $(\mathrm{H}-\sigma \mathrm{l}) \mathbf{w}=$ $\mathrm{LU} \mathbf{w}=\mathbf{u}$, which results in $\mathbf{w}=(\mathrm{H}-\sigma \mathrm{l})^{-1} \mathbf{u}$. We use the shift-and-invert method provided by Spectra library [88] built on top of Eigen library [66]. This algorithm also transforms the matrix inversion to a system of linear equations and has the advantage that it can give arbitrary number of states closest to the target.

\section{APPENDIX B: EFFICIENT ESTIMATION OF THE DENSITY OF STATES}

In order to use the shift-and-invert method, one needs a target eigenvalue. Since we are interested in the eigenstate closest to the maximum density of states, we need to know the location of the maximum of the density of states. If the full eigendecomposition is possible, the density of states is obtained as a trivial side product. However, a more efficient way of estimating the density of states is to apply Sylvester's law of inertia [39], which gives the number of eigenvalues of a matrix $\mathrm{H}$ within an arbitrary interval $\left[\varepsilon_{i}, \varepsilon_{i+1}\right]$.

In our case, $\mathrm{H}$ is a real, symmetric, and nonsingular matrix with a well-defined $L D L$ decomposition

$$
\mathrm{H}=\mathrm{LDL}^{T} \text {, }
$$

where $L$ is a lower unit triangular matrix and $D$ is a diagonal matrix. Sylvester's law of inertia states that the number of positive diagonal entries of the matrix $D$ is equal to the number of positive eigenvalues of the matrix $\mathrm{H}$. One can then construct the shifted matrices $\mathrm{H}-\varepsilon_{i} \mathrm{I}$ and $\mathrm{H}-\varepsilon_{i+1} \mathrm{l}$, whose $L D L$ decompositions give the number of eigenvalues of $\mathrm{H}$ that are larger than $\varepsilon_{i}$ and $\varepsilon_{i+1}$, respectively. The difference of 
these numbers gives the exact number of eigenvalues within the interval $\left[\varepsilon_{i}, \varepsilon_{i+1}\right]$, which can be used to construct the density of states. The algorithm that we use to perform the $L D L$ decomposition is included in Eigen library [66].

A disadvantage of applying Sylvester's law of inertia is the need to perform several full $L D L$ decompositions in order to determine accurately the density of states. This limits the applicability of the method only to moderately small system sizes, $L \lesssim 10$. For larger systems, one has to resort to approximations. Our choice is a method that relies on Chebyshev series [39]. In this method, one considers a projection operator

$$
\hat{P}_{\varepsilon_{i}, \varepsilon_{i+1}}=\sum_{\lambda_{j} \in\left[\varepsilon_{i}, \varepsilon_{i+1}\right]}\left|u_{j}\right\rangle\left\langle u_{j}\right|
$$

constructed from eigenvectors $\left|u_{j}\right\rangle$ whose eigenvalues $\lambda_{j}$ are within the interval $\left[\varepsilon_{i}, \varepsilon_{i+1}\right]$. The trace of this operator then gives exactly the number of eigenvalues within this interval of interest. Since the eigenvectors are not known a priori, one has to approximate both the operator and its trace. Our discussion here follows closely to that given in Ref. [39].

In the eigenbasis of the Hamiltonian matrix $\mathrm{H}$, the projection operator (B2) can be written as

$$
\mathrm{P}_{\varepsilon_{i}, \varepsilon_{i+1}}=\left(\begin{array}{ccccccccc}
\ddots & & & & & & & & \\
& 0 & 0 & 0 & \ldots & 0 & 0 & 0 & \\
& 0 & 1 & 0 & \ldots & 0 & 0 & 0 & \\
& 0 & 0 & 1 & \ldots & 0 & 0 & 0 & \\
& \vdots & \vdots & \vdots & \ddots & \vdots & \vdots & \vdots & \\
& 0 & 0 & 0 & \ldots & 1 & 0 & 0 & \\
0 & 0 & 0 & \ldots & 0 & 1 & 0 & \\
0 & 0 & 0 & \ldots & 0 & 0 & 0 & \\
& & & & & & & \ddots
\end{array}\right) ;
$$

i.e., its diagonals are determined by a boxcar function

$$
P_{\varepsilon_{i}, \varepsilon_{i+1}}^{j j}= \begin{cases}1, & \lambda_{j} \in\left[\varepsilon_{i}, \varepsilon_{i+1}\right] \\ 0, & \text { otherwise }\end{cases}
$$

and trace of the projection matrix thus gives the number of eigenvalues inside the corresponding interval:

$$
\mu_{\varepsilon_{i}, \varepsilon_{i+1}}=\operatorname{Tr}\left(\mathrm{P}_{\varepsilon_{i}, \varepsilon_{i+1}}\right) \text {. }
$$

We interpret the projection matrix as a boxcar function of the Hamiltonian matrix $\mathrm{H}$

$$
\mathrm{P}_{\varepsilon_{i}, \varepsilon_{i+1}}(\mathrm{H})=\mathcal{H}\left(\mathrm{H}-\varepsilon_{i} \mathrm{l}\right)-\mathcal{H}\left(\mathrm{H}-\varepsilon_{i+1} \mathrm{l}\right),
$$

where $\mathcal{H}(x)$ is the Heaviside step function. A boxcar function can be expressed as a series of orthogonal functions. We use the Chebyshev expansion due to its rapid convergence and efficient recursion relations, but in principle one could choose also other basis functions, for example, a Fourier series. We expand the projection matrix as

$$
\begin{aligned}
\mathrm{P}_{\varepsilon_{i}, \varepsilon_{i+1}}(\mathrm{H}) & =\sum_{j=0}^{\infty} \gamma_{j}\left(\varepsilon_{i}, \varepsilon_{i+1}\right) \mathrm{T}_{j}(\mathrm{H}) \\
& \approx \sum_{j=0}^{p} \gamma_{j}\left(\varepsilon_{i}, \varepsilon_{i+1}\right) \mathrm{T}_{j}(\mathrm{H}),
\end{aligned}
$$

where $T_{j}$ is the $j$ th Chebyshev polynomial of the first kind, the series is truncated to the order $p$, and the expansion coefficients $\gamma_{j}\left(\varepsilon_{i}, \varepsilon_{i+1}\right)$ are those of the boxcar function $\gamma_{0}\left(\varepsilon_{i}, \varepsilon_{i+1}\right)=\left[\arccos \left(\varepsilon_{i}\right)-\arccos \left(\varepsilon_{i+1}\right)\right] / \pi$ and for $j \geqslant 1$

$$
\gamma_{j}\left(\varepsilon_{i}, \varepsilon_{i+1}\right)=2 \frac{\sin \left[j \arccos \left(\varepsilon_{i}\right)\right]-\sin \left[j \arccos \left(\varepsilon_{i+1}\right)\right]}{j \pi} .
$$

Here, we have assumed that all the eigenvalues of $\mathrm{H}$ are inside the domain of the Chebyshev polynomials $[-1,1]$. We therefore first have to scale the matrix

$$
\mathrm{H} \rightarrow \frac{\mathrm{H}-\mathrm{I}\left(\lambda_{\max }+\lambda_{\min }\right) / 2}{\mathrm{I}\left(\lambda_{\max }-\lambda_{\min }\right) / 2},
$$

where $\lambda_{\min }$ and $\lambda_{\max }$ are the smallest and largest eigenvalues of $\mathrm{H}$, respectively. Similar scaling has to be done also to the values $\varepsilon_{i}$ and $\varepsilon_{i+1}$.

In principle, one could obtain an estimation for the number of eigenvalues by taking the trace of the sum in Eq. (B7). The problem with this is that in order to construct the value of the matrix-argument Chebyshev polynomial $\mathrm{T}_{p}(\mathrm{H})$, one needs several matrix-matrix multiplications, which makes it a heavy calculation. However, we can reduce the amount of required computational resources considerably if we include the trace operation into Eq. (B7). This replaces the matrix-matrix products with matrix-vector and vector-vector products. An option for performing the trace is to use the full computational basis set in which $\mathrm{H}$ is expressed, but because the matrix $\mathrm{H}$ is large, this is not very efficient. A better approach is to use a Monte-Carlo-like method. We utilize a stochastic estimator by Hutchinson [89], who proved that the trace of a matrix $A$ can be obtained as a stochastic average of random vectors $\mathbf{v}_{k}$ whose elements are either 1 or -1 with equal probabilities: $\operatorname{Tr} A=\lim _{M \rightarrow \infty} M^{-1} \sum_{k=1}^{M} \mathbf{v}_{k}^{T} A \mathbf{v}_{k}$. For our purpose, we write the Hutchinson stochastic trace estimator as

$$
\operatorname{Tr}\left(\mathrm{P}_{\varepsilon_{i}, \varepsilon_{i+1}}\right) \approx \frac{1}{n_{v}} \sum_{k=1}^{n_{v}} \mathbf{v}_{k}^{T} \mathrm{P}_{\varepsilon_{i}, \varepsilon_{i+1}} \mathbf{v}_{k},
$$

where we have truncated the amount of random vectors to $n_{v}$, which is much smaller than the dimension of $\mathrm{H}$. We combine Eqs. (B7) and (B10) and obtain

$$
\mu_{\varepsilon_{i}, \varepsilon_{i+1}} \approx \frac{1}{n_{v}} \sum_{k=1}^{n_{v}} \sum_{j=0}^{p} \gamma_{j}\left(\varepsilon_{i}, \varepsilon_{i+1}\right) \mathbf{v}_{k}^{T} \mathrm{~T}_{j}(\mathrm{H}) \mathbf{v}_{k} .
$$

Let us denote the vector $\mathrm{T}_{j}(\mathrm{H}) \mathbf{v}_{k}$ with $\mathbf{w}_{j}^{k}$. With the recursion relation for the Chebyshev polynomials, one can write this as

$$
\mathbf{w}_{j}^{k}=2 \mathrm{H} \mathbf{w}_{j-1}^{k}-\mathbf{w}_{j-2}^{k},
$$

where, since $T_{0}(H)=I$ and $T_{1}(H)=H$, we have $\mathbf{w}_{0}^{k}=\mathbf{v}_{k}$ and $\mathbf{w}_{1}^{k}=\mathrm{Hv}_{k}$. Finally, Eq. (B11) becomes

$$
\mu_{\varepsilon_{i}, \varepsilon_{i+1}} \approx \frac{1}{n_{v}} \sum_{k=1}^{n_{v}} \sum_{j=0}^{p} \gamma_{j}\left(\varepsilon_{i}, \varepsilon_{i+1}\right) \mathbf{v}_{k}^{T} \mathbf{w}_{j}^{k},
$$

which only contains matrix-vector and vector-vector products. In the Chebyshev expansion, we have used $n_{v}=30$ random 
TABLE II. Comparison of the scaling of the execution times for estimating the number of eigenvalues within an arbitrary interval $\left[\varepsilon_{i}, \varepsilon_{i+1}\right]$ based on the $L D L$ matrix decomposition and the stochastic Chebyshev expansion. $D_{L / 2, L}$ denotes the Hilbert space dimension of Eq. (5) at half-filling for different sized systems $L$. All computations are performed on the same tabletop machine using a single thread from a four-threaded Intel i5-2400 core with $3.10 \mathrm{GHz}$ clock frequency. The execution times of the Chebyshev expansion method scales roughly linearly with the system size due to its sparse matrix-vector multiplication.

\begin{tabular}{lccc}
\hline \hline$L$ & $D_{L / 2, L}$ & $L D L$ decomposition & Chebyshev expansion \\
\hline 10 & 2002 & $0.02 \mathrm{~s}$ & $0.15 \mathrm{~s}$ \\
12 & 12376 & $10 \mathrm{~s}$ & $1.1 \mathrm{~s}$ \\
14 & 77520 & $2500 \mathrm{~s}$ & $4.7 \mathrm{~s}$ \\
16 & 490314 & & $41.0 \mathrm{~s}$ \\
\hline \hline
\end{tabular}

vectors and $p=50$ terms, which are sufficient for accurate results. Performance and accuracy comparisons between the $L D L$ decomposition and the stochastic Chebyshev expansion methods are shown in Table II and Fig. 9, showing that the Chebyshev method produces the maximum of the density of states both rapidly and accurately.

In summary, the desired eigenstate is obtained in a following way. We first create a random realization of on-site energies $\omega_{\ell}$ and construct the Hamiltonian of Eq. (8). If the system is small, we use the exact $L D L$ method to construct the density of states, and otherwise we use the stochastic method of Eq. (B13). We then select the target energy at which the density of states has its maximum. This target energy is then used in the shift-and-invert algorithm to obtain the eigenpair closest to the target. The largest and smallest eigenstates, required in Eq. (B9), can be obtained, e.g., with power iteration or with algorithms included in Spectra [88].

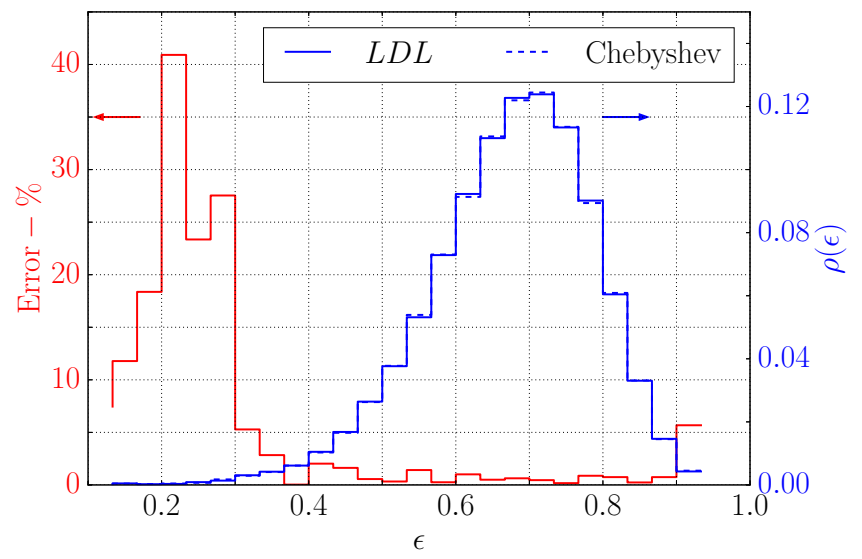

FIG. 9. Comparison of the density of states histograms for system with $L=12$ using $L D L$ matrix decomposition (solid blue) and Chebyshev expansion (dashed blue). Relative error percentage is shown as the red histogram and it tells how much the results obtained with the Chebyshev expansion differ from those obtained with the $L D L$ method. The Chebyshev method is the most accurate in intervals with large number of states. Around the maximum density of states the error is $\approx 1 \%$. In the Chebyshev expansion, we use 50 terms and the stochastic trace is obtained with 30 random vectors.

\section{APPENDIX C: TIME EVOLUTION WITH KRYLOV SUBSPACE METHODS}

The unitary time evolution of a closed quantum system is governed by the Schrödinger equation

$$
i \hbar \frac{d}{d t}|\psi(t)\rangle=\hat{H}|\psi(t)\rangle .
$$

If the Hamiltonian $\hat{H}$ is time independent, the Schrödinger equation has the formal solution

$$
|\psi(t)\rangle=e^{-i t \hat{H} / \hbar}\left|\psi_{0}\right\rangle,
$$

where $\left|\psi_{0}\right\rangle$ is the initial state. If one is able to diagonalize the Hamiltonian, that is, to form the eigendecomposition, the matrix exponential of Eq. (C2) is trivial. However, for large systems, the full diagonalization is inefficient or even impossible, and therefore the matrix exponential has to be approximated. In this work, we use the Krylov subspace method, allowing efficient computation of the product between an exponentiated sparse matrix and a state vector [36,90-93].

If the system at time $t$ is in state $|\psi(t)\rangle$, after a time $\tau$ the state becomes

$$
|\psi(t+\tau)\rangle=e^{-i \tau \hat{H} / \hbar}|\psi(t)\rangle,
$$

If the time step $\tau$ is short, one can accurately express the state vector $|\psi(t)\rangle$ and the Hamiltonian $\hat{H}$ in an $m$-dimensional Krylov subspace $\mathcal{K}_{m}$, with $m$ much smaller than the Hilbert space dimension. This subspace is spanned by the vectors

$$
\left\{\mathbf{v}_{0}, \mathrm{Hv}_{0}, \mathrm{H}^{2} \mathbf{v}_{0}, \ldots, \mathrm{H}^{m-1} \mathbf{v}_{0}\right\},
$$

where the vector $\mathbf{v}_{0}$ denotes the state $|\psi(t)\rangle$ and $\mathrm{H}$ is the Hamiltonian in the matrix form. Because $\mathrm{H}$ is Hermitian, an orthogonal matrix

$$
\mathrm{K}_{m}=\left(\begin{array}{lllll}
\mathbf{v}_{0} & \mathbf{v}_{1} & \mathbf{v}_{2} & \ldots & \mathbf{v}_{m-1}
\end{array}\right)
$$

can be constructed with the Lanczos algorithm [36,90-94]. The Hamiltonian in the Krylov subspace then becomes a tridiagonal $m \times m$ matrix

$$
\mathrm{K}_{m}^{\dagger} \mathrm{HK}_{m}=\mathrm{M}_{m}=\left(\begin{array}{ccccc}
\alpha_{0} & \beta_{1} & & & 0 \\
\beta_{1} & \alpha_{1} & \beta_{2} & & \\
& \beta_{2} & \alpha_{2} & \ddots & \\
& & \ddots & \ddots & \beta_{m-1} \\
0 & & & \beta_{m-1} & \alpha_{m-1}
\end{array}\right) \text {. }
$$

The matrix elements $\alpha_{j}$ and $\beta_{j}$ as well as the orthogonal vectors $\mathbf{v}_{j}$ are obtained from equations [90,93]

$$
\begin{aligned}
\alpha_{j} & =\mathbf{v}_{j} \cdot\left(\mathbf{H}_{j}-\beta_{j-1} \mathbf{v}_{j-1}\right), \\
\beta_{j} \mathbf{v}_{j+1} & =H \mathbf{v}_{j}-\alpha_{j} \mathbf{v}_{j}-\beta_{j-1} \mathbf{v}_{j-1} .
\end{aligned}
$$

After solving the matrix $\mathrm{K}_{m}$, the approximative time evolution can be calculated in the Krylov subspace as [36,91]

$$
|\psi(t+\tau)\rangle=e^{-i \tau \hat{H} / \hbar}|\psi(t)\rangle \approx \mathrm{K}_{m} e^{-i \tau \mathrm{M}_{m} / \hbar} \mathbf{K}_{m}^{\dagger} \mathbf{v}_{0},
$$

where the exponential of the small tridiagonal matrix $\mathrm{M}_{m}$ is easily computed either with the eigendecomposition or Padé 


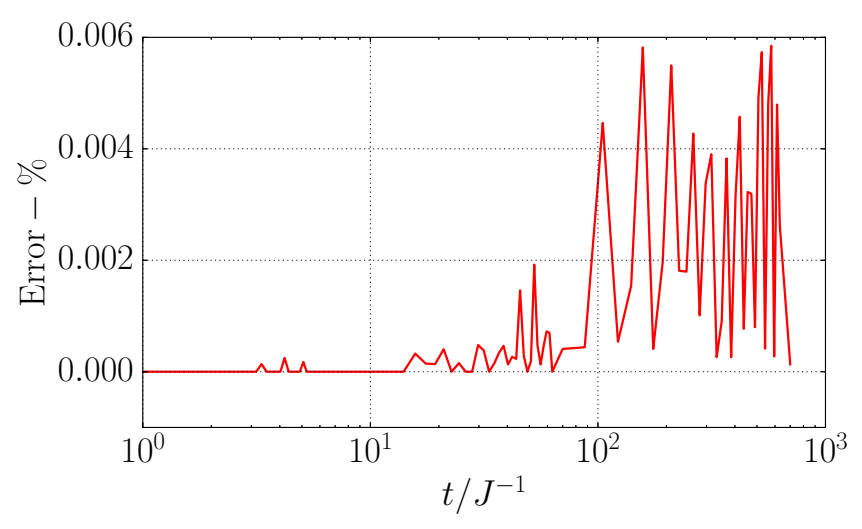

FIG. 10. Relative error percent of the bipartite entanglement entropy obtained from the exact diagonalization and Krylov method with $m=5$ as a function of evolution time calculated for a single, representative disorder realization. The system parameters of Eq. (8) are $L=12, W / J=15$, and $U / J=3.5$.

approximation provided by many numerical libraries. The Krylov method gives accurate results because the eigenvalues of the tridiagonal matrix approximate the eigenvalues of the Hamiltonian that are the most important for the dynamics during the current time step [91].

The Lanczos algorithm used for constructing the basis of the Krylov subspace can suffer from numerical problems. The floating-point arithmetic causes numerical instabilities which lead to a loss of orthogonality of the vectors $\mathbf{v}_{j}$ with increasing subspace dimension $m$ [90,93]. This can be fixed by performing a reorthogonalization on the matrix K. However, for our simulations, the Krylov method produces sufficiently accurate results before the orthogonality is lost. Error of the Krylov method as compared to the exact diagonalization is shown in Fig. 10. We see that the Krylov method is very accurate even for very small subspace size $m=5$. The error is cumulative, which restricts this method to shorter times than the exact diagonalization.

\section{APPENDIX D: TIME EVOLVING BLOCK DECIMATION WITH MATRIX PRODUCT STATES}

For long chains of transmons, we express the wave function $|\psi\rangle$ in terms of matrix product states (MPS) and then utilize an approximate method, denoted as the time-evolving block decimation, to efficiently calculate the unitary time evolution of Eq. (C3). First, matrix product states for an open chain of $L$ transmons are defined as

$$
|\psi\rangle=\sum_{\{n\}} A_{1}^{n_{1}} A_{2}^{n_{2}} \ldots A_{L}^{n_{L}}\left|n_{1} n_{2} \ldots n_{L}\right\rangle,
$$

where the index $n_{\ell} \in 0,1, \ldots, n_{\max }$ labels the number of bosons at site $\ell$ with maximum (truncated) occupation $n_{\max }$ and the sum is performed over all "physical" indices $n_{\ell}$. $A_{\ell}^{n_{\ell}}$ is a $N_{\ell} \times M_{\ell}$ matrix. For the left-right boundary tensors, $N_{1}, M_{L}=1$. The dimensions associated to $N_{\ell}$ and $M_{\ell}$ are the "bond" dimensions between sites $\ell-1, \ell$ and $\ell, \ell+1$, respectively. In numerical algorithms, we bound the size of the matrix $N_{\ell}, M_{\ell}<\mathcal{D}$. The bound on bond dimension, for

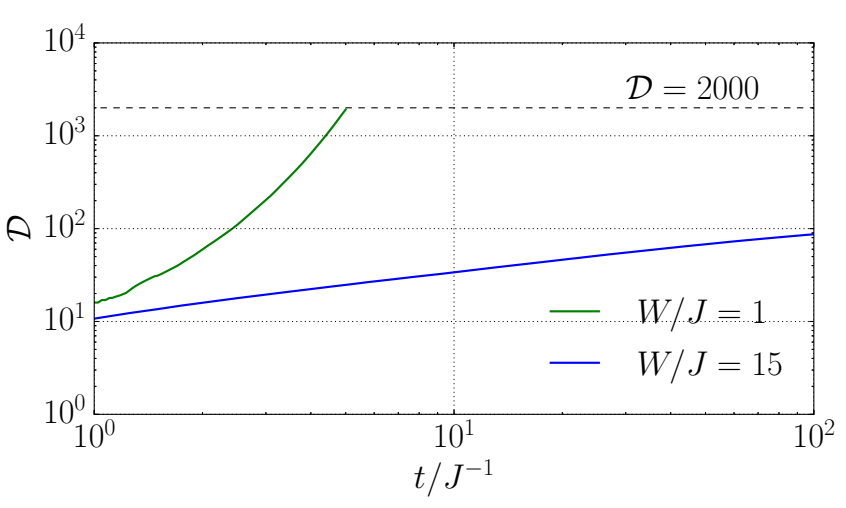

FIG. 11. Evolution of the average bond dimension $\mathcal{D}$ in ergodic (green) and many-body localized (blue) phases.

example $N_{\ell}$, is equivalent to a bound on entanglement entropy $S \sim \ln \mathcal{D}$, across the bipartition $(1, \ell-1) \cup(\ell, L)$.

The total number of resources $\mathcal{R}$ required to store a matrix product state is $\mathcal{R} \sim n_{\max } \mathcal{D}^{2} L$. If the entanglement entropy is small, matrix product states provide an efficient classical storage container of many-body quantum states. Examples of such states are gapped ground states, many-body localized eigenstates and in general any state which obeys area law in entanglement entropy, i.e., $S \sim$ const for all bipartitions $(1, \ell-1) \cup(\ell, L)$. Unfortunately, entanglement entropy for a quench at finite-energy density in a chaotic system increases linearly with time $S \propto t$, which implies that for a fixed cutoff $\mathcal{D} \sim \mathcal{O}\left(10^{3}\right)$ the state can be quantitatively approximated up to times for which $T \propto \ln (\mathcal{D})$. On the other hand, for many-body localized systems $S \propto \ln t$, which implies $T \propto$ $\mathcal{D}^{c}$, where $c$ is a disorder dependent constant. In Fig. 11, we compare the computational resources over time in the ergodic and many-body localized phases by monitoring the growth of average bond dimension over time. We track the largest bond dimension per disorder realization and then average over realizations. As expected, the scaling in the localized phase is a power law $\mathcal{D}_{\text {loc }} \propto t^{a}$, while the scaling in the ergodic phase is exponential $\mathcal{D}_{\text {erg }} \propto e^{a t}$.

Time evolving block decimation [37] is an evolution scheme based on the application of the Trotter formula to the unitary time evolution, i.e., breaking the many-body unitary to a successive application of few-body unitary gates, and a controlled truncation of the matrix product state after the application of each gate.

\section{Integrator}

We first describe the integrator we use, which is a fourthorder Trotterization scheme. For a nearest-neighbor Hamiltonian, we define the "forward-backward" sweeps (timeevolution steps) as

$$
\begin{aligned}
& \Omega(d t)=U_{L, L-1}(d t) U_{L-1, L-2}(d t), \ldots, U_{2,1}(d t), \\
& \Omega^{*}(d t)=U_{1,2}(d t) U_{2,3}(d t), \ldots, U_{L-1, L}(d t),
\end{aligned}
$$


where the two-site gates are defined as

$$
U_{\ell, \ell+1}(d t)=e^{-i h_{\ell, \ell+1} d t}
$$

The Hamiltonian density $h_{\ell, \ell+1}$ is defined to symmetrically include the one-site terms of the Hamiltonian of Eq. (8),

$$
\begin{aligned}
h_{\ell, \ell+1} & =\frac{1}{2}\left(h_{\ell}+h_{\ell+1}\right)+J_{\ell}\left(\hat{a}_{\ell}^{\dagger} \hat{a}_{\ell+1}+\text { H.c. }\right), \\
h_{\ell} & =\omega_{\ell} \hat{n}_{\ell}-\frac{U_{\ell}}{2} \hat{n}_{\ell}\left(\hat{n}_{\ell}-1\right) .
\end{aligned}
$$

The backward sweep is the adjoint method of the forward sweep, i.e., $\Omega^{*}(-d t) \Omega(d t)|\psi\rangle=|\psi\rangle$. It can be shown that for self-adjoint methods the Trotter error is always of even order [95]. Thus, it is favorable to create the self-adjoint method $\Psi(d t)=\Omega^{*}(d t / 2) \Omega(d t / 2)$, which is a second-order method. Using standard composition rules [95], we compose the following fourth-order self-adjoint method,

$$
F(d t)=\Psi\left(a_{1} d t\right) \Psi\left(a_{2} d t\right) \Psi\left(a_{1} d t\right)
$$

where $\quad a_{1}=\left(2-2^{1 / 3}\right)^{-1}, \quad a_{2}=1-2 a_{1}, \quad$ and $F(T)=e^{-i H T / \hbar}+T \mathcal{O}\left(d t^{4}\right)$.

\section{Truncation}

Matrix product states have some gauge freedom associated to the virtual bond dimension. We use the "mixed" gauge [96] to perform the evolution and efficiently truncate the matrix product state. We discuss the process for a forward sweep. Before applying the gate $U_{\ell, \ell+1}$ mixed gauge implies that local tensors satisfy

$$
\begin{aligned}
& \sum_{n_{k}} A_{k}^{n_{k} \dagger} A_{k}^{n_{k}}=\mathbb{1}_{M_{k} \times M_{k}}, k<\ell, \\
& \sum_{n_{k}} A_{k}^{n_{k}} A_{k}^{n_{k} \dagger}=\mathbb{1}_{N_{k} \times N_{k}}, k>\ell,
\end{aligned}
$$

while the tensor at site $\ell$ does not have any special property. The tensors at site $\ell, \ell+1$ are combined and the two-body gate is applied,

$$
\Theta_{\ell, \ell+1}=U_{\ell, \ell+1} \sum_{n_{\ell}, n_{\ell+1}} A_{\ell}^{n_{\ell}} A_{\ell+1}^{n_{\ell+1}}\left|n_{\ell} n_{\ell+1}\right\rangle .
$$

The updated local tensors are constructed from the singular value decomposition,

$$
\begin{aligned}
\Theta_{\left(n_{\ell} b_{\ell}\right),\left(n_{\ell+1} c_{\ell+1}\right)} & =\sum_{a} \mathcal{U}_{\left(n_{\ell} b_{\ell}\right), a} S_{a, a} \mathcal{V}_{a,\left(n_{\ell+1} c_{\ell+1}\right)}^{*}, \\
A_{b_{\ell}, a_{\ell}}^{n_{\ell}} & =\mathcal{U}_{\left(n_{\ell} b_{\ell}\right), a}, \\
A_{a^{\prime}, c_{\ell+1}}^{n_{\ell+1}} & =\sum_{a} S_{a^{\prime}, a} \mathcal{V}_{a,\left(n_{\ell+1} c_{\ell+1}\right)}^{*},
\end{aligned}
$$

Because of the unitarity of the matrix $\mathcal{U}, \mathcal{U}^{\dagger} \mathcal{U}=11$, the updated tensor $A_{\ell}$ obeys the correct gauge form required for the evolution of sites of $\ell+1, \ell+2$. The singular values $\lambda_{k}$ (in descending order) are the diagonal elements of $S$ and correspond to the square roots of the eigenvalues of the reduced density matrix $\rho=\operatorname{Tr}_{1 \ldots \ell}|\psi\rangle\langle\psi|$, thus $\sum_{k} \lambda_{k}^{2}=1$. We truncate the singular values for $k>k_{c}$ so that the probability loss $\sum_{k>k_{c}} \lambda_{k}^{2}<\epsilon$, and then renormalize the truncated density matrix, $\sum_{k \leqslant k_{c}} \lambda_{k}^{2}=1$. This process is iterated to perform a forward sweep. The backward sweep is performed in a similar way. The computational bottleneck of the algorithm is the singular value decomposition of Eq. (D8), which scales as $\mathcal{O}\left[\left(n_{\max } \mathcal{D}\right)^{3}\right]$ for a square matrix of dimension $n_{\max } \mathcal{D}$.

\section{Numerical details}

Calculations were performed using the ITensor Library [97]. In all simulations, we have explicitly conserved the $U$ (1) symmetry associated to the total particle number, which allows for an additional speedup due to the block diagonal structure of the Eq. (D7). For the simulations in the ergodic regime $(W / J=1, U / J=3.5)$, we have used time step $d t / J^{-1}=0.025$ and cutoff $\epsilon=10^{-8}$ as well as a hard cutoff to the bond dimension $\mathcal{D}_{c}=2000$. The local Hilbert space is truncated at $n_{\max }=4$. The simulations are stopped at time $T / J^{-1}=5$, where the hard cutoff is reached for most realizations to ensure that the quantities of interest are accurate. For the disordered regime $(W / J=15, U / J=3.5)$, we have used $d t / J^{-1}=0.01$ and cutoff $\epsilon=10^{-9}$ as well as a hard cutoff to the bond dimension $\mathcal{D}_{c}=2000$. To gain additional speedup, we truncated the local Hilbert space at $n_{\max }=3$ which does not affect the dynamics for strong disorder and dilute initial state. The simulations are stopped at time $T / \mathrm{J}^{-1}=$ 200. The bond dimension is not saturated for any disorder realization.
[1] D. L. Underwood, W. E. Shanks, J. Koch, and A. A. Houck, Low-disorder microwave cavity lattices for quantum simulation with photons, Phys. Rev. A 86, 023837 (2012).

[2] J. Koch, T. M. Yu, J. Gambetta, A. A. Houck, D. I. Schuster, J. Majer, A. Blais, M. H. Devoret, S. M. Girvin, and R. J. Schoelkopf, Charge-insensitive qubit design derived from the Cooper pair box, Phys. Rev. A 76, 042319 (2007).

[3] Y. Chen, C. Neill, P. Roushan, N. Leung, M. Fang, R. Barends, J. Kelly, B. Campbell, Z. Chen, B. Chiaro, A. Dunsworth, E. Jeffrey, A. Megrant, J. Y. Mutus, P. J. J. O’Malley, C. M. Quintana, D. Sank, A. Vainsencher, J. Wenner, T. C. White,
M. R. Geller, A. N. Cleland, and J. M. Martinis, Qubit Architecture with High Coherence and Fast Tunable Coupling, Phys. Rev. Lett. 113, 220502 (2014).

[4] R. Nandkishore and D. A. Huse, Many-body localization and thermalization in quantum statistical mechanics, Annu. Rev. Condens. Matter Phys. 6, 15 (2015).

[5] F. Alet and N. Laflorencie, Many-body localization: An introduction and selected topics, C. R. Phys. 19, 498 (2018).

[6] D. A. Abanin, E. Altman, I. Bloch, and M. Serbyn, Colloquium: Many-body localization, thermalization, and entanglement, Rev. Mod. Phys. 91, 021001 (2019). 
[7] M. Serbyn, Z. Papić, and D. A. Abanin, Universal Slow Growth of Entanglement in Interacting Strongly Disordered Systems, Phys. Rev. Lett. 110, 260601 (2013).

[8] L. D’Alessio, Y. Kafri, A. Polkovnikov, and M. Rigol, From quantum chaos and eigenstate thermalization to statistical mechanics and thermodynamics, Adv. Phys. 65, 239 (2016).

[9] S. Choi, J. Choi, R. Landig, G. Kucsko, H. Zhou, J. Isoya, F. Jelezko, S. Onoda, H. Sumiya, V. Khemani, C. von Keyserlingk, N. Y. Yao, E. Demler, and M. D. Lukin, Observation of discrete time-crystalline order in a disordered dipolar many-body system, Nature (London) 543, 221 (2017).

[10] J. Zhang, P. W. Hess, A. Kyprianidis, P. Becker, A. Lee, J. Smith, G. Pagano, I.-D. Potirniche, A. C. Potter, A. Vishwanath, N. Y. Yao, and C. Monroe, Observation of a discrete time crystal, Nature (London) 543, 217 (2017).

[11] K. Sacha and J. Zakrzewski, Time crystals: A review, Rep. Prog. Phys. 81, 016401 (2018).

[12] V. Oganesyan and D. A. Huse, Localization of interacting fermions at high temperature, Phys. Rev. B 75, 155111 (2007).

[13] Y. Bar Lev and D. R. Reichman, Dynamics of many-body localization, Phys. Rev. B 89, 220201(R) (2014).

[14] Y. Bar Lev, G. Cohen, and D. R. Reichman, Absence of Diffusion in an Interacting System of Spinless Fermions on a One-Dimensional Disordered Lattice, Phys. Rev. Lett. 114, 100601 (2015).

[15] D. J. Luitz, N. Laflorencie, and F. Alet, Many-body localization edge in the random-field Heisenberg chain, Phys. Rev. B 91, 081103(R) (2015).

[16] R. Singh, J. H. Bardarson, and F. Pollmann, Signatures of the many-body localization transition in the dynamics of entanglement and bipartite fluctuations, New J. Phys. 18, 023046 (2016).

[17] A. Pal and D. A. Huse, Many-body localization phase transition, Phys. Rev. B 82, 174411 (2010).

[18] A. Nanduri, H. Kim, and D. A. Huse, Entanglement spreading in a many-body localized system, Phys. Rev. B 90, 064201 (2014).

[19] M. Žnidarič, T. Prosen, and P. Prelovšek, Many-body localization in the Heisenberg $X X Z$ magnet in a random field, Phys. Rev. B 77, 064426 (2008).

[20] L. F. Santos and M. Rigol, Localization and the effects of symmetries in the thermalization properties of one-dimensional quantum systems, Phys. Rev. E 82, 031130 (2010).

[21] I. L. Aleiner, B. L. Altshuler, and G. V. Shlyapnikov, A finitetemperature phase transition for disordered weakly interacting bosons in one dimension, Nat. Phys. 6, 900 (2010).

[22] P. Sierant, D. Delande, and J. Zakrzewski, Many-body localization for randomly interacting bosons, Acta Phys. Pol. A 132, 1707 (2017)

[23] P. Sierant and J. Zakrzewski, Many-body localization of bosons in optical lattices, New J. Phys. 20, 043032 (2018).

[24] P. Sierant, K. Biedroń, G. Morigi, and J. Zakrzewski, Manybody localization in presence of cavity mediated long-range interactions, SciPost Phys. 7, 008 (2019).

[25] M. Pino, L. B. Lev, and B. L. Altshuler, Nonergodic metallic and insulating phases of Josephson junction chains, P. Natl. Acad. Sci. USA 113, 536 (2016).

[26] P. Sierant, D. Delande, and J. Zakrzewski, Many-body localization due to random interactions, Phys. Rev. A 95, 021601(R) (2017).
[27] P. Roushan, C. Neill, J. Tangpanitanon, V. M. Bastidas, A. Megrant, R. Barends, Y. Chen, Z. Chen, B. Chiaro, A. Dunsworth, A. Fowler, B. Foxen, M. Giustina, E. Jeffrey, J. Kelly, E. Lucero, J. Mutus, M. Neeley, C. Quintana, D. Sank, A. Vainsencher, J. Wenner, T. White, H. Neven, D. G. Angelakis, and J. Martinis, Spectroscopic signatures of localization with interacting photons in superconducting qubits, Science $\mathbf{3 5 8}$, 1175 (2017).

[28] C. Neill, P. Roushan, K. Kechedzhi, S. Boixo, S. V. Isakov, V. Smelyanskiy, A. Megrant, B. Chiaro, A. Dunsworth, K. Arya, R. Barends, B. Burkett, Y. Chen, Z. Chen, A. Fowler, B. Foxen, M. Giustina, R. Graff, E. Jeffrey, T. Huang, J. Kelly, P. Klimov, E. Lucero, J. Mutus, M. Neeley, C. Quintana, D. Sank, A. Vainsencher, J. Wenner, T. C. White, H. Neven, and J. M. Martinis, A blueprint for demonstrating quantum supremacy with superconducting qubits, Science 360, 195 (2018).

[29] S. Hacohen-Gourgy, V. V. Ramasesh, C. De Grandi, I. Siddiqi, and S. M. Girvin, Cooling and Autonomous Feedback in a Bose-Hubbard Chain with Attractive Interactions, Phys. Rev. Lett. 115, 240501 (2015).

[30] R. Ma, B. Saxberg, C. Owens, N. Leung, Y. Lu, J. Simon, and D. I. Schuster, A dissipatively stabilized Mott insulator of photons, Nature (London) 566, 51 (2019).

[31] J.-y. Choi, S. Hild, J. Zeiher, P. Schauß, A. Rubio-Abadal, T. Yefsah, V. Khemani, D. A. Huse, I. Bloch, and C. Gross, Exploring the many-body localization transition in two dimensions, Science 352, 1547 (2016).

[32] M. Rispoli, A. Lukin, R. Schittko, S. Kim, M. E. Tai, J. Léonard, and M. Greiner, Quantum critical behavior at the many-bodylocalization transition, Nature (London) 573, 385 (2019).

[33] A. Lukin, M. Rispoli, R. Schittko, M. E. Tai, A. M. Kaufman, S. Choi, V. Khemani, J. Léonard, and M. Greiner, Probing entanglement in a many-body-localized system, Science 364, 256 (2019).

[34] M. Dalmonte, S. I. Mirzaei, P. R. Muppalla, D. Marcos, P. Zoller, and G. Kirchmair, Realizing dipolar spin models with arrays of superconducting qubits, Phys. Rev. B 92, 174507 (2015).

[35] T. Brydges, A. Elben, P. Jurcevic, B. Vermersch, C. Maier, B. P. Lanyon, P. Zoller, R. Blatt, and C. F. Roos, Probing Rényi entanglement entropy via randomized measurements, Science 364, 260 (2019).

[36] D. J. Luitz and Y. B. Lev, The ergodic side of the many-body localization transition, Ann. Phys. 529, 1600350 (2017).

[37] G. Vidal, Efficient Simulation of One-Dimensional Quantum Many-Body Systems, Phys. Rev. Lett. 93, 040502 (2004).

[38] B. Pirvu, V. Murg, J. I. Cirac, and F. Verstraete, Matrix product operator representations, New J. Phys. 12, 025012 (2010).

[39] E. D. Napoli, E. Polizzi, and Y. Saad, Efficient estimation of eigenvalue counts in an interval, Numer. Linear Algebra Appl. 23, 674 (2016).

[40] H. Paik, D. I. Schuster, L. S. Bishop, G. Kirchmair, G. Catelani, A. P. Sears, B. R. Johnson, M. J. Reagor, L. Frunzio, L. I. Glazman, S. M. Girvin, M. H. Devoret, and R. J. Schoelkopf, Observation of High Coherence in Josephson Junction Qubits Measured in a Three-Dimensional Circuit QED Architecture, Phys. Rev. Lett. 107, 240501 (2011).

[41] K. Xu, J.-J. Chen, Y. Zeng, Y.-R. Zhang, C. Song, W. Liu, Q. Guo, P. Zhang, D. Xu, H. Deng, K. Huang, H. Wang, X. Zhu, D. Zheng, and H. Fan, Emulating Many-Body Localization with 
a Superconducting Quantum Processor, Phys. Rev. Lett. 120, 050507 (2018).

[42] C. Song, K. Xu, H. Li, Y. Zhang, X. Zhang, W. Liu, Q. Guo, Z. Wang, W. Ren, J. Hao, H. Feng, H. Fan, D. Zheng, D. Wang, H. Wang, and S. Zhu, Observation of multi-component atomic Schrödinger cat states of up to 20 qubits, Science 365, 574 (2019)

[43] J. Chang, M. R. Vissers, A. D. Corcoles, M. Sandberg, J. Gao, D. W. Abraham, J. M. Chow, J. M. Gambetta, M. B. Rothwell, G. A. Keefe, M. Steffen, and D. P. Pappas, Improved superconducting qubit coherence using titanium nitride, App. Phys. Lett. 103, 012602 (2013).

[44] R. Barends, J. Kelly, A. Megrant, A. Veitia, D. Sank, E. Jeffrey, T. C. White, J. Mutus, A. G. Fowler, B. Campbell, Y. Chen, Z. Chen, B. Chiaro, A. Dunsworth, C. Neill, P. O'Malley, P. Roushan, A. Vainsencher, J. Wenner, A. N. Korotkov, A. N. Cleland, and J. M. Martinis, Superconducting quantum circuits at the surface code threshold for fault tolerance, Nature (London) 508, 500 (2014).

[45] C. Rigetti, J. M. Gambetta, S. Poletto, B. L. T. Plourde, J. M. Chow, A. D. Córcoles, J. A. Smolin, S. T. Merkel, J. R. Rozen, G. A. Keefe, M. B. Rothwell, M. B. Ketchen, and M. Steffen, Superconducting qubit in a waveguide cavity with a coherence time approaching $0.1 \mathrm{~ms}$, Phys. Rev. B 86, 100506(R) (2012).

[46] G. Wendin, Quantum information processing with superconducting circuits: A review, Rep. Prog. Phys. 80, 106001 (2017).

[47] J. M. Gambetta, J. M. Chow, and M. Steffen, Building logical qubits in a superconducting quantum computing system, npj Quantum Inf. 3, 2 (2017).

[48] M. J. Peterer, S. J. Bader, X. Jin, F. Yan, A. Kamal, T. J. Gudmundsen, P. J. Leek, T. P. Orlando, W. D. Oliver, and S. Gustavsson, Coherence and Decay of Higher Energy Levels of a Superconducting Transmon Qubit, Phys. Rev. Lett. 114, 010501 (2015).

[49] J. Koch, A. A. Houck, K. L. Hur, and S. M. Girvin, Timereversal-symmetry breaking in circuit-QED-based photon lattices, Phys. Rev. A 82, 043811 (2010).

[50] M. H. Devoret and R. J. Schoelkopf, Superconducting circuits for quantum information: An outlook, Science 339, 1169 (2013).

[51] A. A. Houck, H. E. Türeci, and J. Koch, On-chip quantum simulation with superconducting circuits, Nat. Phys. 8, 292 (2012)

[52] L. Sun, A. Petrenko, Z. Leghtas, B. Vlastakis, G. Kirchmair, K. M. Sliwa, A. Narla, M. Hatridge, S. Shankar, J. Blumoff, L. Frunzio, M. Mirrahimi, M. H. Devoret, and R. J. Schoelkopf, Tracking photon jumps with repeated quantum non-demolition parity measurements, Nature (London) 511, 444 (2014).

[53] N. Ofek, A. Petrenko, R. Heeres, P. Reinhold, Z. Leghtas, B. Vlastakis, Y. Liu, L. Frunzio, S. M. Girvin, L. Jiang, M. Mirrahimi, M. H. Devoret, and R. J. Schoelkopf, Extending the lifetime of a quantum bit with error correction in superconducting circuits, Nature (London) 536, 441 (2016).

[54] B. Skinner, J. Ruhman, and A. Nahum, Measurement-Induced Phase Transitions In the Dynamics of Entanglement, Phys. Rev. X 9, 031009 (2018).

[55] Y. Li, X. Chen, and M. P. A. Fisher, Quantum Zeno effect and the many-body entanglement transition, Phys. Rev. B 98, 205136 (2018).
[56] A. Chan, R. M. Nandkishore, M. Pretko, and G. Smith, Unitaryprojective entanglement dynamics, Phys. Rev. B 99, 224307 (2019).

[57] Y. Li, X. Chen, and M. P. A. Fisher, Measurementdriven entanglement transition in hybrid quantum circuits, arXiv:1901.08092 (unpublished).

[58] S. Choi, Y. Bao, X.-L. Qi, and E. Altman, Quantum error correction and entanglement phase transition in random unitary circuits with projective measurements, arXiv:1903.05124 (unpublished).

[59] M. J. Gullans and D. A. Huse, Dynamical purification phase transition induced by quantum measurements, arXiv:1905.05195 (unpublished).

[60] M. Hatridge, S. Shankar, M. Mirrahimi, F. Schackert, K. Geerlings, T. Brecht, K. M. Sliwa, B. Abdo, L. Frunzio, S. M. Girvin, R. J. Schoelkopf, and M. H. Devoret, Quantum backaction of an individual variable-strength measurement, Science 339, 178 (2013).

[61] R. Lescanne, L. Verney, Q. Ficheux, M. H. Devoret, B. Huard, M. Mirrahimi, and Z. Leghtas, Escape of a Driven Quantum Josephson Circuit Into Unconfined States, Phys. Rev. Appl. 11, 014030 (2019).

[62] L. Verney, R. Lescanne, M. H. Devoret, Z. Leghtas, and M. Mirrahimi, Structural Instability of Driven Josephson Circuits Prevented By an Inductive Shunt, Phys. Rev. Appl. 11, 024003 (2019).

[63] I. Pietikäinen, J. Tuorila, D. S. Golubev, and G. S. Paraoanu, Photon blockade and the quantum-to-classical transition in the driven-dissipative Josephson pendulum coupled to a resonator, Phys. Rev. A 99, 063828 (2019).

[64] M. P. A. Fisher, P. B. Weichman, G. Grinstein, and D. S. Fisher, Boson localization and the superfluid-insulator transition, Phys. Rev. B 40, 546 (1989).

[65] F. Pietracaprina, N. Macé, D. J. Luitz, and F. Alet, Shift-invert diagonalization of large many-body localizing spin chains, SciPost Phys. 5, 045 (2018).

[66] Eigen, ver. 3, http://eigen.tuxfamily.org.

[67] M. Fitzpatrick, N. M. Sundaresan, A. C. Y. Li, J. Koch, and A. A. Houck, Observation of a Dissipative Phase Transition in a One-Dimensional Circuit QED Lattice, Phys. Rev. X 7, 011016 (2017).

[68] R. Mondaini and M. Rigol, Many-body localization and thermalization in disordered Hubbard chains, Phys. Rev. A 92 041601(R) (2015).

[69] J. M. Deutsch, Quantum statistical mechanics in a closed system, Phys. Rev. A 43, 2046 (1991).

[70] M. Srednicki, Chaos and quantum thermalization, Phys. Rev. E 50, 888 (1994).

[71] P. W. Anderson, Absence of diffusion in certain random lattices, Phys. Rev. 109, 1492 (1958).

[72] J. A. Miszczak, Singular value decomposition and matrix reorderings in quantum information theory, Int. J. Mod. Phys. C 22, 897 (2011).

[73] H. F. Song, S. Rachel, C. Flindt, I. Klich, N. Laflorencie, and K. Le Hur, Bipartite fluctuations as a probe of many-body entanglement, Phys. Rev. B 85, 035409 (2012).

[74] Y. Y. Atas, E. Bogomolny, O. Giraud, and G. Roux, Distribution of the Ratio of Consecutive level Spacings in Random Matrix Ensembles, Phys. Rev. Lett. 110, 084101 (2013). 
[75] K. Kudo and T. Deguchi, Finite-size scaling with respect to interaction and disorder strength at the many-body localization transition, Phys. Rev. B 97, 220201(R) (2018).

[76] M. V. Medvedyeva, T. Prosen, and M. Žnidarič, Influence of dephasing on many-body localization, Phys. Rev. B 93, 094205 (2016).

[77] E. Levi, M. Heyl, I. Lesanovsky, and J. P. Garrahan, Robustness of Many-Body Localization in the Presence of Dissipation, Phys. Rev. Lett. 116, 237203 (2016).

[78] H. P. Lüschen, P. Bordia, S. S. Hodgman, M. Schreiber, S. Sarkar, A. J. Daley, M. H. Fischer, E. Altman, I. Bloch, and U. Schneider, Signatures of Many-Body Localization in a Controlled Open Quantum System, Phys. Rev. X 7, 011034 (2017).

[79] J. H. Bardarson, F. Pollmann, and J. E. Moore, Unbounded Growth of Entanglement in Models of Many-Body Localization, Phys. Rev. Lett. 109, 017202 (2012).

[80] M. Goihl, M. Friesdorf, A. H. Werner, W. Brown, and J. Eisert, Experimentally accessible witnesses of many-body localisation, Quantum Rep. 1, 50 (2019).

[81] H. Kim and D. A. Huse, Ballistic Spreading of Entanglement in a Diffusive Nonintegrable System, Phys. Rev. Lett. 111, 127205 (2013).

[82] M. Serbyn, Z. Papić, and D. A. Abanin, Quantum quenches in the many-body localized phase, Phys. Rev. B 90, 174302 (2014).

[83] G. De Tomasi, F. Pollmann, and M. Heyl, Solving efficiently the dynamics of many-body localized systems at strong disorder, Phys. Rev. B 99, 241114 (2018).

[84] P. Krantz, M. Kjaergaard, F. Yan, T. P. Orlando, S. Gustavsson, and W. D. Oliver, A quantum engineer's guide to superconducting qubits, Appl. Phys. Rev. 6, 021318 (2019).

[85] E. H. Lieb and D. W. Robinson, The finite group velocity of quantum spin systems, in Statistical Mechanics (Springer, Berlin, 1972), pp. 425-431.
[86] S. Johri, R. Nandkishore, and R. N. Bhatt, Many-Body Localization in Imperfectly Isolated Quantum Systems, Phys. Rev. Lett. 114, 117401 (2015).

[87] M. H. Fischer, M. Maksymenko, and E. Altman, Dynamics of a Many-Body-Localized System Coupled to a Bath, Phys. Rev. Lett. 116, 160401 (2016).

[88] Spectra, https://spectralib.org/.

[89] M. Hutchinson, A stochastic estimator of the trace of the influence matrix for Laplacian smoothing splines, Commun. Stat. Simulat. 19, 433 (1990).

[90] Y. Saad, Iterative Methods for Sparse Linear Systems, 2nd ed. (Society for Industrial and Applied Mathematics, Philadelphia, 2003), pp. 194-196.

[91] C. Moler and C. Van Loan, Nineteen dubious ways to compute the exponential of a matrix, twenty-five years later, SIAM Rev. 45, 3 (2003).

[92] S. R. Manmana, A. Muramatsu, and R. M. Noack, Time evolution of one-dimensional quantum many body systems, in Lectures on the Physics of Highly Correlated Electron Systems IX: Ninth Training Course in the Physics of Correlated Electron Systems and High-Tc Superconductors, AIP Conf. Proc. No. 789 (AIP, New York, 2005), p. 269.

[93] R. Beerwerth and H. Bauke, Krylov subspace methods for the Dirac equation, Comput. Phys. Commun. 188, 189 (2015).

[94] C. Lanczos, An iteration method for the solution of the eigenvalue problem of linear differential and integral operators, J. Res. Nat. Bur. Stand. 45, 255 (1950).

[95] J. E. Marsden, S. S. Antman, L. Wiggins, S. Sirovich, L. Glass, R. V. Kohn, and S. S. Sastry, Interdisciplinary Applied Mathematics (Springer, Berlin, 1993), Vol. 3.

[96] U. Schollwöck, The density-matrix renormalization group in the age of matrix product states, Ann. Phys. 326, 96 (2011).

[97] ITensor Library, ver. 2, http://itensor.org. 